\title{
Dietary Nitrates, Nitrites, and Nitrosamines Intake and the Risk of Gastric Cancer: A Meta-Analysis
}

\author{
Peng Song ${ }^{1,+}$, Lei $\mathrm{Wu}^{2,+}$ and Wenxian Guan ${ }^{1, *}$ \\ Received: 22 September 2015; Accepted: 16 November 2015; Published: 1 December 2015 \\ 1 Department of General Surgery, Nanjing Drum Tower Hospital, The Affiliated Hospital of Nanjing \\ University Medical School, Nanjing 210008, China; pengsong90@126.com \\ 2 Department of Laboratory Medicine, The First Affiliated Hospital of Nanjing Medical University, \\ Nanjing 210029, China; leiwu90@gmail.com \\ * Correspondence: wenxian_guan@126.com; Tel.: +25-681-82098 \\ + These authors contributed equally to this work.
}

\begin{abstract}
The potential associations between dietary consumption of nitrates, nitrites, and nitrosamines and gastric cancer risk have been investigated by several studies, but yielded inconclusive results. We conducted a meta-analysis to provide a quantitative assessment of their relationships. Relevant articles were identified by a systematic literature searching of PubMed and Embase databases prior to August 2015. Random-effects models were employed to pool the relative risks. A total of 22 articles consisting of 49 studies-19 studies for nitrates, 19 studies for nitrites, and 11 studies for $N$-nitrosodimethylamine (NDMA) - were included. The summary relative risk of stomach cancer for the highest categories, compared with the lowest, was 0.80 (95\% confidence interval (CI), 0.69-0.93) for dietary nitrates intake, 1.31 (95\% CI, 1.13-1.52) for nitrites, and 1.34 (95\% CI, 1.02-1.76) for NDMA ( $p$ for heterogeneity was $0.015,0.013$ and $<0.001$, respectively). The study type was found as the main source of heterogeneity for nitrates and nitrites. The heterogeneity for NDMA could not be eliminated completely through stratified analysis. Although significant associations were all observed in case-control studies, the cohort studies still showed a slight trend. The dose-response analysis indicated similar results as well. High nitrates intake was associated with a weak but statistically significant reduced risk of gastric cancer. Whereas increased consumption of nitrites and NDMA seemed to be risk factors for cancer. Due to the lack of uniformity for exposure assessment across studies, further prospective researches are warranted to verify these findings.
\end{abstract}

Keywords: diet; nitrates; nitrites; nitrosamines; gastric cancer

\section{Introduction}

Over recent decades, the incidence and mortality rate of gastric cancer showed a modest decline globally. In 2012, an estimated almost one million new stomach cancer cases and 700,000 deaths occurred, making it the fifth most common malignancy and the third leading cause of cancer related deaths worldwide [1]. Nearly half of the world's new cases occurred in Eastern Asia, especially China. Geographic differences were observed in gastric cancer incidence, indicating that some modifiable factors (e.g., diet) could play a critical role in the etiology of this malignancy [2,3]. Therefore, it is an urgent demand to identify risk factors that can have a marked impact on this disease.

The typical diet in most countries contains nitrates, nitrites, and nitrosamines. Nitrates and nitrites occur naturally in fruit and vegetables, which are regarded as an important part of a healthy diet due to the powerful evidence of beneficial health effects against cancer [4,5]. In the same time, nitrates and nitrites are often used as food additives in processed meats such as ham, bacon, sausages, 
and hot dogs, to retard microbial spoilage, and preserve meat products recognizable appearance and flavor as well. A high consumption of processed meats is linked to an increased gastric cancer risk, and many people consider nitrates/nitrites as the main reason for that [6]. Nitrosamines are produced by chemical reactions of nitrates, nitrites and other proteins. $N$-nitrosodimethylamine (NDMA) is one of the most frequently occurring nitrosamines in our dietary foods $[7,8]$. NDMA is a potent carcinogen, capable of inducing malignant tumors in various animal species in a variety of tissues, including liver, lung, and stomach $[9,10]$.

So far, numerous epidemiologic studies have been published which attempted to assess the potential risk of gastric cancer about the dietary nitrates, nitrites, and nitrosamines intake, but yielded discrepant findings. It would be of interest to evaluate, on the basis of current epidemiologic data, whether consumption of nitrates, nitrites, and NDMA had an effect on gastric carcinogenesis. To clarify their relations, we systematically reviewed all the available evidence and conducted a meta-analysis.

\section{Methods}

\subsection{Literature Search and Selection}

We searched PubMed and Embase databases through August 2015 using the following search terms: (1) nitrate AND (gastric cancer OR stomach cancer); (2) nitrite AND (gastric cancer OR stomach cancer); and (3) (nitrosamine OR N-nitrosodimethylamine OR NDMA) AND (gastric cancer OR stomach cancer). The manual search was supplemented by scrutinizing the reference lists from those retrieved articles to identify any relevant studies.

For inclusion, the study must meet the following criteria: (1) cohort or case-control design; (2) exposure of interest was dietary nitrates, nitrites, and NDMA intake; (3) the endpoint of interest was gastric cancer; (4) risk estimates with corresponding 95\% confidence intervals (95\% CIs) were provided; (5) published in English. When study populations were overlapped or duplicated in some studies, we chose the most complete and suitable research. Three authors evaluated the retrieved literature and any discrepancy was resolved through discussion.

\subsection{Data Extraction and Quality Assessment}

Two investigators using a standardized form to extract the following characteristics independently: first author's name, publication year, population information, study location and period, sample size, follow-up years, nitrates and nitrites and NDMA intake assessment, and relative risk (RR)/hazard ratio (HR)/odds ratio (OR) with 95\% CI from the most fully adjusted model for each category.

Quality of the included studies was assessed using Newcastle-Ottawa Scale (NOS) with a score ranging from 0 to 9 [11]. Each study was evaluated based on three aspects: selection, comparability, and assessment of outcome or ascertainment of exposure. Studies with score $\geqslant 7$ were defined as being of high quality.

\subsection{Statistical Analysis}

RR was used to measure the association between the dietary nitrates, nitrites, and NDMA intake and the risk of stomach cancer. Because the absolute risk of gastric cancer was low, OR and HR approximated the RR [12]. Considering the variations within-study and between-study, a random-effects model was employed to calculate the summary RR by pooling each study risk estimate. Statistical heterogeneity among studies was assessed with the $\chi^{2}$-based $\mathrm{Q}$ and $I^{2}$ index. If three or more studies were available for the same characteristic, subgroup analyses were conducted.

For the dose-response analysis, we used the method proposed by Orsini et al. to calculate the risk trend [13]. This method required the number of case and control subjects, or cases and person-years, and median level of dietary nitrates or nitrites or NDMA intake for at least three quantitative exposure 
categories. The median or mean consumption of each category was assigned to the corresponding dose of consumption. We assumed that open-ended category had the same amplitude as the ahead or behind category. Potential nonlinear association was assessed using restricted cubic splines with four knots at percentiles $5 \%, 35 \%, 65 \%$, and $95 \%$ of the distribution. If linear dose-response regression with no heterogeneity was detected, we used it directly.

Meta-regression was employed to explore the possible heterogeneity, and study design, geographic area, and publication year were examined in the model. We also undertook sensitivity analysis to evaluate whether a single study could affect the overall outcome. Publication bias was assessed by funnel plot, with Egger's regression asymmetry test and Begg's adjusted rank correlation test, and the Duval and Tweedie "trim and fill" method was performed if bias was detected [14]. All analyses were completed using STATA version 12.0 (Stata, College Station, TX, USA). A two-sided $p<0.05$ was considered statistically significant.

\subsection{Disease Assessment and Dietary Assessment}

As indicated above, the scope of this meta-analysis was the association between dietary nitrates/nitrites/NDMA intake and stomach cancer. The cases should be confirmed with reliable medical records such as surgical, pathology reports or linkage of authoritative tumor registries. The methods of exposure ascertainment will be extracted, which could vary considerably by the following factors: estimates from various food items and based on different food composition databases.

\section{Results}

\subsection{Literature Search and Quality Assessment}

Based on the search strategy, a total of 22 articles consisting of 49 studies were included in our meta-analysis (Figure 1). Of the 22 papers, there were seven prospective cohort studies and 15 case-control studies. Among them, 15 eligible articles (19 studies) were retrieved for nitrates, 14 articles (19 studies) analyzed nitrites, and eight articles (11 studies) focused on NDMA. Tables 1 and 2 showed the detailed characteristics of these studies. Most of the studies were carried out in North America and Europe. The publication years were from 1985 to 2013. The sample size ranged from 220 to 494,979 and the number of gastric cancer patients varied from 79 to 1016. Methods of dietary exposure differed across studies. Briefly, all included studies generally used a questionnaire to assess dietary nitrates/nitrites/NDMA intake, and that was computed by multiplying the frequency of intake of each unit of food item by the nutrient content values from food composition databases. Studies included met quality criteria of 6-9 stars (Supplemental Table S1 and Supplemental Table S2). 
Table 1. Characteristics of prospective cohort studies in the meta-analysis.

\begin{tabular}{|c|c|c|c|c|c|c|c|c|c|}
\hline $\begin{array}{l}\text { First } \\
\text { Author, } \\
\text { Year, } \\
\text { Location }\end{array}$ & $\begin{array}{l}\text { Cohort } \\
\text { Size }\end{array}$ & $\begin{array}{l}\text { Follow-up } \\
\text { (Years) }\end{array}$ & $\begin{array}{l}\text { No. of Cases } \\
\text { (Age/Definition) }\end{array}$ & Intake Assessment & $\begin{array}{l}\text { Analytical } \\
\text { Category }\end{array}$ & $\begin{array}{l}\text { Definition/Nutrient } \\
\text { Content Values }\end{array}$ & $\begin{array}{l}\text { Consumption } \\
\text { Categories }\end{array}$ & $\begin{array}{l}\text { Adjusted RR } \\
(95 \% \text { CI })\end{array}$ & Adjusted Variables \\
\hline $\begin{array}{l}\text { Galanis, } \\
1998 \text { [15], } \\
\text { Hawaii }\end{array}$ & $\begin{array}{l}5610 \\
\text { men } \\
\text { and } \\
6297 \\
\text { women }\end{array}$ & 14.8 & $\begin{array}{l}108 \text { (NA/form Hawaii } \\
\text { Tumor Registry, a member } \\
\text { of the Surveillance, } \\
\text { Epidemiology, and End } \\
\text { Results (SEER) program } \\
\text { of the National Cancer } \\
\text { Institute) }\end{array}$ & $\begin{array}{l}\text { short questionnarire } \\
\text { (weekly frequency } \\
\text { of intake of } 13 \text { foods } \\
\text { and food groups, } \\
\text { and the daily } \\
\text { frequency of intake } \\
\text { of } 6 \text { beverages) }\end{array}$ & $\begin{array}{l}\text { Nitrates } \\
\text { times/week }\end{array}$ & $\begin{array}{l}\text { combined frequency of } \\
\text { intake of dried fish, pickled } \\
\text { vegetables and processed } \\
\text { meats/based on previous } \\
\text { published literature }\end{array}$ & $\begin{array}{l}0-3 \\
4-7 \\
\geqslant 8\end{array}$ & $\begin{array}{l}1.0 \text { (Referent) } \\
1.30(0.80-2.00) \\
0.90(0.50-1.40)\end{array}$ & $\begin{array}{l}\text { Age, years of } \\
\text { education, Japanese } \\
\text { place of birth, gender. } \\
\text { Analyses among men } \\
\text { were also adjusted for } \\
\text { cigarette smoking and } \\
\text { alcohol intake status }\end{array}$ \\
\hline \multirow{7}{*}{$\begin{array}{l}\text { Van } \\
\text { Loon, } \\
1998 \text { [16], } \\
\text { the } \\
\text { Netherlands }\end{array}$} & \multirow{7}{*}{$\begin{array}{l}1688 \\
\text { men } \\
\text { and } \\
1812 \\
\text { women }\end{array}$} & \multirow{7}{*}{6.3} & \multirow{7}{*}{$\begin{array}{l}282 \text { (mean: } 63.0 \text { years, SD: } \\
4.1 \text { /exclude cases } \\
\text { self-reported, in situ } \\
\text { carcinoma, or without } \\
\text { microscopically } \\
\text { confirmed diagnosis) }\end{array}$} & \multirow{7}{*}{$\begin{array}{l}\text { 150-item } \\
\text { semiquantitative } \\
\text { FFQ }\end{array}$} & \multirow{7}{*}{$\begin{array}{l}\begin{array}{l}\text { Nitrates } \\
\text { mg/day }\end{array} \\
\\
\begin{array}{l}\text { Nitrites } \\
\text { mg/day }\end{array}\end{array}$} & \multirow{7}{*}{$\begin{array}{l}\text { derived from vegetables } \\
\text { (considered loss during } \\
\text { preparation) and drinking } \\
\text { water/from State Institute } \\
\text { for Quality Control of } \\
\text { Agricultural Products solely } \\
\text { on the intake of cured } \\
\text { meat/from TNO Nutrition } \\
\text { and Food Research Institute }\end{array}$} & 59.8 & 1.0 (Referent) & \multirow{7}{*}{$\begin{array}{l}\text { Age, sex, smoking, } \\
\text { education, coffee } \\
\text { consumption, intake } \\
\text { of vitamin C and } \\
\text { beta-carotene, family } \\
\text { history of stomach } \\
\text { cancer, prevalence of } \\
\text { stomach disorders, } \\
\text { use of refrigerator or } \\
\text { freezer }\end{array}$} \\
\hline & & & & & & & 84.7 & $1.25(0.84-1.86)$ & \\
\hline & & & & & & & 127.3 & $0.92(0.59-1.44)$ & \\
\hline & & & & & & & 0.01 & 1.0 (Referent) & \\
\hline & & & & & & & 0.09 & $1.18(0.77-1.82)$ & \\
\hline & & & & & & & 0.16 & $0.88(0.56-1.37)$ & \\
\hline & & & & & & & 0.35 & $1.44(0.95-2.18)$ & \\
\hline
\end{tabular}


Table 1. Cont.

\begin{tabular}{|c|c|c|c|c|c|c|c|c|c|}
\hline $\begin{array}{l}\text { First } \\
\text { Author, } \\
\text { Year, } \\
\text { Location }\end{array}$ & $\begin{array}{l}\text { Cohort } \\
\text { Size }\end{array}$ & $\begin{array}{l}\text { Follow-up } \\
\text { (Years) }\end{array}$ & $\begin{array}{l}\text { No. of Cases } \\
\text { (Age/Definition) }\end{array}$ & Intake Assessment & $\begin{array}{l}\text { Analytical } \\
\text { Category }\end{array}$ & $\begin{array}{l}\text { Definition/Nutrient Content } \\
\text { Values }\end{array}$ & $\begin{array}{l}\text { Consumption } \\
\text { Categories }\end{array}$ & $\begin{array}{l}\text { Adjusted RR } \\
(95 \% \text { CI })\end{array}$ & Adjusted Variables \\
\hline \multirow{3}{*}{$\begin{array}{l}\text { Knekt, } \\
1999 \text { [17], } \\
\text { Finnish }\end{array}$} & \multirow{3}{*}{$\begin{array}{l}9985 \\
\text { men } \\
\text { and } \\
\text { women }\end{array}$} & \multirow{3}{*}{24} & \multirow{3}{*}{$\begin{array}{l}68 \text { (15-49 years: } 18 \text { cases, } \\
50-59 \text { years: } 28, \text { cases, } \\
60-99 \text { years: } 22 \\
\text { cases / through the } \\
\text { nationwide Finnish } \\
\text { Cancer Registry) }\end{array}$} & \multirow[t]{3}{*}{$\begin{array}{l}\text { pre-formed } \\
\text { qeustionnaire }\end{array}$} & $\begin{array}{l}\text { Nitrates } \\
\text { Quartiles }\end{array}$ & $\begin{array}{l}\text { derived from vegetables } \\
(91.9 \%) / \text { based on foods tables } \\
\text { in Finland and other countries } \\
\text { in northern Europe }\end{array}$ & $\begin{array}{l}\text { Q1 } \\
\text { Q2 } \\
\text { Q3 } \\
\text { Q4 }\end{array}$ & $\begin{array}{l}1.0 \text { (Referent) } \\
1.01(0.56-1.84) \\
0.52(0.25-1.08) \\
0.56(0.27-1.18)\end{array}$ & \multirow{3}{*}{$\begin{array}{l}\text { Age, sex, } \\
\text { municipality, } \\
\text { smoking and } \\
\text { energy intake }\end{array}$} \\
\hline & & & & & \multirow[b]{2}{*}{$\begin{array}{l}\text { NDMA } \\
\text { Quartiles }\end{array}$} & $\begin{array}{l}\text { derived mainly from cured } \\
\text { meats and sausages } \\
(94.2 \%) / \text { based on foods tables } \\
\text { in Finland and other countries } \\
\text { in northern Europe }\end{array}$ & $\begin{array}{l}\text { Q1 } \\
\text { Q2 } \\
\text { Q3 } \\
\text { Q4 }\end{array}$ & $\begin{array}{l}1.0 \text { (Referent) } \\
1.10(0.58-2.11) \\
1.88(1.01-3.49) \\
0.71(0.28-1.78)\end{array}$ & \\
\hline & & & & & & $\begin{array}{l}\text { derived from smoked and salted } \\
\text { fish }(51.9 \%), \text { cured meats and } \\
\text { susages }(48.1 \%) / \text { based on foods } \\
\text { tables in Finland and other } \\
\text { countries in northern Europe }\end{array}$ & $\begin{array}{l}\text { Q1 } \\
\text { Q2 } \\
\text { Q3 } \\
\text { Q4 }\end{array}$ & $\begin{array}{l}1.0 \text { (Referent) } \\
1.03(0.55-1.95) \\
0.78(0.39-1.56) \\
0.75(0.37-1.51)\end{array}$ & \\
\hline $\begin{array}{l}\text { Jakszyn, } \\
2006 \text { [8], } \\
\text { European }\end{array}$ & $\begin{array}{l}153,447 \\
\text { men } \\
\text { and } \\
368,010 \\
\text { women }\end{array}$ & 6.6 & $\begin{array}{l}314 \text { (mean: } 59.2 \text { years, SD: } \\
7.48 / \text { confirmed by a } \\
\text { panel of pathologists) }\end{array}$ & $\begin{array}{l}\text { country-specific } \\
\text { validated } \\
\text { questionnaires }\end{array}$ & $\begin{array}{l}\text { NDMA } \\
\text { Tertiles }\end{array}$ & $\begin{array}{l}\text { matched food items on the } \\
\text { country-specific questionnaires } \\
\text { with a food database of } \\
\text { potential carcinogens/based on } \\
\text { country-specific values }\end{array}$ & $\begin{array}{l}\text { T1 } \\
\text { T2 } \\
\text { T3 }\end{array}$ & $\begin{array}{l}1.0 \text { (Referent) } \\
0.87 \text { (0.64-1.20) } \\
0.99(0.69-1.41) \\
\text { Cardia } \\
1.0 \text { (Referent) } \\
0.74(0.41-1.34) \\
0.68 \text { (0.34-1.37) } \\
\text { Non-cardia } \\
1.0 \text { (Referent) } \\
1.04 \text { (0.66-1.63) } \\
1.09(0.65-1.81)\end{array}$ & $\begin{array}{l}\text { Full cohort analysis: } \\
\text { stratified center and } \\
\text { age. Sex, height, } \\
\text { weight, education } \\
\text { level, tobacco } \\
\text { smoking, cigarette } \\
\text { smoking intensity, } \\
\text { work and leisure } \\
\text { physical activity, } \\
\text { citrus and } \\
\text { non-citrus fruits } \\
\text { intake, vegetables } \\
\text { intake, energy } \\
\text { intake and nitrites }\end{array}$ \\
\hline
\end{tabular}


Table 1. Cont

\begin{tabular}{|c|c|c|c|c|c|c|c|c|c|}
\hline $\begin{array}{l}\text { First } \\
\text { Author, } \\
\text { Year, } \\
\text { Location }\end{array}$ & $\begin{array}{l}\text { Cohort } \\
\text { Size }\end{array}$ & $\begin{array}{l}\text { Follow-up } \\
\text { (Years) }\end{array}$ & $\begin{array}{l}\text { No. of Cases } \\
\text { (Age/Definition) }\end{array}$ & Intake Assessment & $\begin{array}{l}\text { Analytical } \\
\text { Category }\end{array}$ & $\begin{array}{l}\text { Definition/Nutrient } \\
\text { Content Values }\end{array}$ & $\begin{array}{l}\text { Consumption } \\
\text { Categories }\end{array}$ & $\begin{array}{l}\text { Adjusted RR } \\
(95 \% \text { CI })\end{array}$ & Adjusted Variables \\
\hline $\begin{array}{l}\text { Larsson, } \\
2006 \text { [18], } \\
\text { Sweden }\end{array}$ & $\begin{array}{l}61,433 \\
\text { women }\end{array}$ & 18 & $\begin{array}{l}156 \text { (NA/through linkage } \\
\text { of the study population } \\
\text { with the national and } \\
\text { regional Swedish Cancer } \\
\text { registers) }\end{array}$ & $\begin{array}{l}\text { 67-item FFQ (before } \\
\text { 1997) 97-item FFQ } \\
\text { (after 1997) }\end{array}$ & $\begin{array}{l}\text { NDMA } \\
\mu \mathrm{g} / \text { day }\end{array}$ & $\begin{array}{l}\text { estimated by matching } \\
\text { questionnaire food } \\
\text { items/based on foods tables } \\
\text { on the Swedish market }\end{array}$ & $\begin{array}{l}<0.041 \\
0.041-0.078 \\
0.079-0.120 \\
0.121-0.193 \\
\geqslant 0.194\end{array}$ & $\begin{array}{l}1.0 \text { (Referent) } \\
1.03(0.61-1.77) \\
1.66(1.00-2.75) \\
1.60(0.93-2.76) \\
1.96(1.08-3.58) \\
\end{array}$ & $\begin{array}{l}\text { Age, education, } \\
\text { body mass index, } \\
\text { intakes of total } \\
\text { energy, alcohol, } \\
\text { fruits and } \\
\text { vegetables }\end{array}$ \\
\hline $\begin{array}{l}\text { Cross, } \\
2011[19], \\
\text { USA }\end{array}$ & $\begin{array}{l}295,305 \\
\text { men } \\
\text { and } \\
199,674 \\
\text { women }\end{array}$ & 10 & $\begin{array}{l}532 \text { (NA/through } \\
\text { probabilistic linkage with } \\
\text { state cancer registries) }\end{array}$ & 124-item FFQ & $\begin{array}{l}\text { Nitrates } \\
\mu \mathrm{g} / 1000 \mathrm{kcals}\end{array}$ & $\begin{array}{l}\text { derived from processed } \\
\text { meats/using a database of } \\
\text { measured values from ten } \\
\text { types of processed meats in } \\
\text { US }\end{array}$ & $\begin{array}{l}24.2 \\
66.9 \\
112.7 \\
174.5 \\
298.0\end{array}$ & $\begin{array}{l}\text { Cardia } \\
1.0 \text { (Referent) } \\
1.17 \text { (0.77-1.77) } \\
0.64 \text { (0.40-1.02) } \\
0.94 \text { (0.61-1.45) } \\
0.81 \text { (0.52-1.25) } \\
\text { Non-cardia } \\
1.0 \text { (Referent) } \\
0.90 \text { (0.60-1.35) } \\
0.89 \text { (0.59-1.33) } \\
0.91 \text { (0.61-1.37) } \\
1.04 \text { (0.69-1.55) } \\
\text { Cardia } \\
1.0 \text { (Referent) } \\
0.72 \text { (0.47-1.11) } \\
0.88 \text { (0.58-1.32) } \\
0.87 \text { (0.58-1.31) } \\
0.71 \text { (0.47-1.08) } \\
\text { Non-cardia } \\
1.0 \text { (Referent) } \\
0.77 \text { (0.51-1.15) } \\
0.79 \text { (0.53-1.18) } \\
1.04 \text { (0.71-1.52) } \\
0.93 \text { (0.63-1.37) }\end{array}$ & $\begin{array}{l}\text { Age, education, sex, } \\
\text { BMI, ethnicity, } \\
\text { smoking, alcohol } \\
\text { drinking, physical } \\
\text { activity daily intake } \\
\text { of fruits, } \\
\text { vegetables, } \\
\text { saturated fat and } \\
\text { calories }\end{array}$ \\
\hline
\end{tabular}


Table 1. Cont.

\begin{tabular}{|c|c|c|c|c|c|c|c|c|c|}
\hline $\begin{array}{l}\text { First } \\
\text { Author, } \\
\text { Year, } \\
\text { Location }\end{array}$ & $\begin{array}{l}\text { Cohort } \\
\text { Size }\end{array}$ & $\begin{array}{l}\text { Follow-up } \\
\text { (Years) }\end{array}$ & $\begin{array}{l}\text { No. of Cases } \\
\text { (Age/Definition) }\end{array}$ & $\begin{array}{l}\text { Intake } \\
\text { Assessment }\end{array}$ & $\begin{array}{l}\text { Analytical } \\
\text { Category }\end{array}$ & $\begin{array}{l}\text { Definition/Nutrient } \\
\text { Content Values }\end{array}$ & $\begin{array}{l}\text { Consumption } \\
\text { Categories }\end{array}$ & $\begin{array}{l}\text { Adjusted RR } \\
(95 \% \text { CI })\end{array}$ & Adjusted Variables \\
\hline \multirow[t]{2}{*}{$\begin{array}{l}\text { Keszei, } \\
2013 \text { [20], } \\
\text { the } \\
\text { Netherlands }\end{array}$} & \multirow[t]{2}{*}{$\begin{array}{l}120,852 \\
\text { men } \\
\text { and } \\
\text { women }\end{array}$} & \multirow[t]{2}{*}{16.3} & $\begin{array}{l}663 \text { (Women, Cardia, } \\
\text { mean: } 62.6 \text { years, SD: } 4.2 \text {; } \\
\text { Women, Non-cardia, } \\
\text { mean: } 62.6 \text { years, SD: } 4.3 \text {; } \\
\text { Men, Cardia, mean: } 61.4 \\
\text { years, SD: } 4.1 \text {; Men, } \\
\text { Non-cardia, mean: } 62.4 \\
\text { years, SD: } 4.0 / \text { through } \\
\text { linkage to the }\end{array}$ & $\begin{array}{l}\text { questionnaire } \\
\text { including } 150 \\
\text { items on food }\end{array}$ & $\begin{array}{l}\text { Nitrates } \\
\text { Tertiles } \\
\text { (mg/day) }\end{array}$ & $\begin{array}{l}\text { derived from summing } \\
\text { dietary intake (considered } \\
\text { loss during preparation) } \\
\text { and nitrate from } \\
\text { water/based on databank of } \\
\text { the State Institute for } \\
\text { Quality Control of } \\
\text { Agricultural Products }\end{array}$ & $\begin{array}{l}\text { T1: women } \\
66.4 ; \text { men, } \\
68.1 \\
\text { T2: women, } \\
\text { 98.5; men } \\
\text { 100.8 } \\
\text { T3: women } \\
\text { 142.7; men } \\
\text { 146.2 }\end{array}$ & $\begin{array}{l}\text { Women, Cardia } \\
1.0 \text { (Referent) } \\
1.01 \text { (0.30-3.42) } \\
1.61(0.32-8.06) \\
\text { Women, } \\
\text { Non-cardia } \\
1.0 \text { (Referent) } \\
0.73 \text { (0.47-1.11) } \\
0.78 \text { (0.44-1.39) } \\
\text { Men, Cardia } \\
1.0 \text { (Referent) } \\
1.06 \text { (0.68-1.65) } \\
1.01 \text { (0.57-1.77) } \\
\text { Men, } \\
\text { Non-cardia } \\
1.0 \text { (Referent) } \\
1.23 \text { (0.90-1.68) } \\
1.05 \text { (0.70-1.59) }\end{array}$ & $\begin{array}{l}\text { Age, smoking status, } \\
\text { years of cigarette } \\
\text { smoking, number of } \\
\text { cigarettes smoked per } \\
\text { day, total energy } \\
\text { intake, BMI, alcoholic } \\
\text { intake, vegetable } \\
\text { intake, fruit intake, } \\
\text { level of education, } \\
\text { and nonoccupational } \\
\text { physical activity }\end{array}$ \\
\hline & & & $\begin{array}{l}\text { Netherlands Cancer } \\
\text { Registry and the } \\
\text { Nationwide Network and } \\
\text { Registry of Histo- and } \\
\text { Cytopathology in the } \\
\text { Netherlands) }\end{array}$ & & $\begin{array}{l}\text { Nitrites } \\
\text { Tertiles } \\
\text { (mg/day) }\end{array}$ & $\begin{array}{l}\text { processed meat/based on } \\
\text { analyses conducted by the } \\
\text { National Public Health } \\
\text { Institute in } 1984\end{array}$ & $\begin{array}{l}\text { T1: women, } \\
0.02 ; \text { men } \\
0.03 \\
\text { T2: women, } \\
0.08 ; \text { men } \\
0.12 \\
\text { T3: women, } \\
0.20 ; \text { men } \\
0.28\end{array}$ & $\begin{array}{l}\text { Women, Cardia } \\
1.0 \text { (Referent) } \\
0.97 \text { (0.36-2.58) } \\
0.62 \text { (0.20-1.90) } \\
\text { Women, } \\
\text { Non-cardia } \\
1.0 \text { (Referent) } \\
0.94 \text { (0.62-1.41) } \\
1.08 \text { (0.71-1.63) } \\
\text { Men, Cardia } \\
1.0 \text { (Referent) } \\
0.80 \text { (0.51-1.27) } \\
1.18 \text { (0.75-1.86) } \\
\text { Men, } \\
\text { Non-cardia } \\
1.0 \text { (Referent) } \\
1.10 \text { (0.80-1.50) } \\
1.23 \text { (0.89-1.70) }\end{array}$ & $\begin{array}{l}\text { Age, smoking status, } \\
\text { years of cigarette } \\
\text { smoking, number of } \\
\text { cigarettes smoked per } \\
\text { day, total energy } \\
\text { intake, BMI, alcoholic } \\
\text { intake, vegetable } \\
\text { intake, fruit intake, } \\
\text { level of education, } \\
\text { and nonoccupational } \\
\text { physical activity }\end{array}$ \\
\hline
\end{tabular}


Table 1. Cont

\begin{tabular}{|c|c|c|c|c|c|c|c|c|}
\hline $\begin{array}{l}\text { First } \\
\text { Author, } \\
\text { Year, } \\
\text { Location }\end{array}$ & $\begin{array}{l}\text { Cohort } \\
\text { Size }\end{array}$ & $\begin{array}{ll}\text { Follow-up No. of Cases } \\
\text { (Years) } & \text { (Age/Definition) }\end{array}$ & $\begin{array}{l}\text { Intake } \\
\text { Assessment }\end{array}$ & $\begin{array}{l}\text { Analytical } \\
\text { Category }\end{array}$ & $\begin{array}{l}\text { Definition/Nutrient } \\
\text { Content Values }\end{array}$ & $\begin{array}{l}\text { Consumption } \\
\text { Categories }\end{array}$ & $\begin{array}{l}\text { Adjusted RR } \\
(95 \% \text { CI })\end{array}$ & Adjusted Variables \\
\hline & & & & $\begin{array}{l}\text { NDMA } \\
\text { Tertiles } \\
\text { ( } \mu \text { g/day) }\end{array}$ & $\begin{array}{l}N \text {-nitrosodimethylamine } \\
\text { values in food items } \\
\text { together with the frequency } \\
\text { of consumption and serving } \\
\text { sizes/N-nitrosodimethylamine } \\
\text { value for food items used in } \\
\text { the Netherlands Cohort } \\
\text { Study }\end{array}$ & $\begin{array}{l}\text { T1: women, } \\
0.03 \text {; men } \\
0.04 \\
\text { T2: women, } \\
0.04 \text {; men } \\
0.08 \\
\text { T3: women, } \\
0.07 \text {; men } \\
0.25\end{array}$ & $\begin{array}{l}\text { Women, Cardia } \\
1.0 \text { (Referent) } \\
0.97 \text { (0.34-2.78) } \\
1.02(0.33-3.14) \\
\text { Women, } \\
\text { Non-cardia } \\
1.0 \text { (Referent) } \\
1.37 \text { (0.92-2.02) } \\
0.90 \text { (0.58-1.42) } \\
\text { Men, Cardia } \\
1.0 \text { (Referent) } \\
1.00 \text { (0.64-1.56) } \\
0.94 \text { (0.59-1.49) } \\
\text { Men, } \\
\text { Non-cardia } \\
1.0 \text { (Referent) } \\
1.09 \text { (0.79-1.50) } \\
1.31 \text { (0.95-1.81) }\end{array}$ & $\begin{array}{l}\text { Age, smoking status, } \\
\text { years of cigarette } \\
\text { smoking, number of } \\
\text { cigarettes smoked per } \\
\text { day, total energy } \\
\text { intake, BMI, alcoholic } \\
\text { beverages not } \\
\text { including beer, } \\
\text { vegetable intake, fruit } \\
\text { intake, level of } \\
\text { education, and } \\
\text { nonoccupational } \\
\text { physical activity. }\end{array}$ \\
\hline
\end{tabular}

NDMA: N-nitrosodimethylamine; RR: relative risk; CI: confidence interval; BMI: body mass index; FFQ: food frequency questionnaire; SD: standard error; NA: Not Applicable. 
Table 2. Characteristics of case-control studies in the meta-analysis.

\begin{tabular}{|c|c|c|c|c|c|c|c|c|c|}
\hline $\begin{array}{l}\text { First Author, } \\
\text { Year, Location }\end{array}$ & $\begin{array}{l}\text { No. of Cases } \\
\text { (Age/Definition) }\end{array}$ & $\begin{array}{l}\text { No. and Type } \\
\text { of Controls }\end{array}$ & $\begin{array}{l}\text { Study } \\
\text { Period }\end{array}$ & $\begin{array}{l}\text { Intake } \\
\text { Assessment }\end{array}$ & $\begin{array}{l}\text { Analytical } \\
\text { Category }\end{array}$ & $\begin{array}{l}\text { Definition/Nutrient } \\
\text { Content Values }\end{array}$ & $\begin{array}{l}\text { Consumption } \\
\text { Categories }\end{array}$ & $\begin{array}{l}\text { Adjusted OR } \\
(95 \% \text { CI })\end{array}$ & $\begin{array}{l}\text { Adjusted } \\
\text { Variables }\end{array}$ \\
\hline \multirow{4}{*}{$\begin{array}{l}\text { Risch, } 1985 \text { [21], } \\
\text { Canada }\end{array}$} & \multirow{4}{*}{$\begin{array}{l}246 \text { ( } 35-79 \text { years/by } \\
\text { province-wide } \\
\text { tumor registries, and } \\
\text { surgical, pathology, } \\
\text { and medical records) }\end{array}$} & \multirow{4}{*}{$\begin{array}{l}246 \\
\text { population- } \\
\text { based }\end{array}$} & \multirow{4}{*}{ 1979-1982 } & \multirow{4}{*}{$\begin{array}{l}\text { diet frequent } \\
\text { questionnaire }\end{array}$} & $\begin{array}{l}\text { Nitrates } \\
\text { mg/day }\end{array}$ & \multirow{4}{*}{$\begin{array}{l}\text { estimated by matching } \\
\text { FFQ food items/food } \\
\text { composition tables were } \\
\text { modified and extended } \\
\text { to Canadian items }\end{array}$} & \multirow{4}{*}{ NA } & 1.0 (Referent) & \multirow{4}{*}{ NA } \\
\hline & & & & & & & & & \\
\hline & & & & & Nitrites & & & 1.0 (Referent) & \\
\hline & & & & & $\mathrm{mg} /$ day & & & $1.71(1.24-2.37)$ & \\
\hline \multirow{10}{*}{$\begin{array}{l}\text { Buiatti, } 1990 \\
\text { [22], Italy }\end{array}$} & \multirow{10}{*}{$\begin{array}{l}1016(\leqslant 75 \\
\text { years/histologic } \\
\text { confirmation) }\end{array}$} & \multirow{10}{*}{$\begin{array}{l}1159 \\
\text { population- } \\
\text { based }\end{array}$} & \multirow{10}{*}{ 1985-1987 } & \multirow{10}{*}{$\begin{array}{l}\text { 146-item } \\
\text { questionnaire }\end{array}$} & & \multirow{10}{*}{$\begin{array}{l}\text { estimated by matching } \\
\text { questionnaire food } \\
\text { items/using several } \\
\text { Italian sources }\end{array}$} & 53 & 1.0 (Referent) & \multirow{10}{*}{$\begin{array}{l}\text { Non-dietary } \\
\text { variables and } \\
\text { kilocalories }\end{array}$} \\
\hline & & & & & & & 81 & $0.90(0.70-1.10)$ & \\
\hline & & & & & $\begin{array}{l}\text { Nitrates } \\
\mathrm{mg} / \text { day }\end{array}$ & & 103 & $0.90(0.60-1.10)$ & \\
\hline & & & & & & & 130 & $0.70(0.50-0.90)$ & \\
\hline & & & & & & & 193 & $0.90(0.70-1.20)$ & \\
\hline & & & & & \multirow{5}{*}{$\begin{array}{l}\text { Nitrites } \\
\text { mg/day }\end{array}$} & & 2.1 & 1.0 (Referent) & \\
\hline & & & & & & & 2.8 & $1.00(0.80-1.40)$ & \\
\hline & & & & & & & 3.4 & $1.20(0.90-1.70)$ & \\
\hline & & & & & & & 4.1 & $1.40(1.00-2.00)$ & \\
\hline & & & & & & & 5.9 & $1.90(1.30-2.70)$ & \\
\hline \multirow{5}{*}{$\begin{array}{l}\text { Boeing, } 1991 \\
\text { [23], Germany }\end{array}$} & \multirow{5}{*}{$\begin{array}{l}143 \text { ( } 32-80 \text { years / } \\
\text { histologically } \\
\text { confirmed) }\end{array}$} & \multirow{5}{*}{$\begin{array}{l}579 \text { hospital- } \\
\text { based }\end{array}$} & \multirow{5}{*}{ 1985-1988 } & \multirow{5}{*}{$\begin{array}{l}\text { 74-item } \\
\text { standardized } \\
\text { questionnaire }\end{array}$} & \multirow{5}{*}{$\begin{array}{l}\text { Nitrates } \\
\text { Quintiles }\end{array}$} & \multirow{5}{*}{$\begin{array}{l}\text { estimated by matching } \\
\text { questionnaire food } \\
\text { items/German Federal } \\
\text { Agency of Nutrition }\end{array}$} & Q1 & 1.0 (Referent) & \multirow{5}{*}{$\begin{array}{l}\text { Age, sex, and } \\
\text { hospital }\end{array}$} \\
\hline & & & & & & & Q2 & $0.93(0.53-1.64)$ & \\
\hline & & & & & & & Q3 & $0.61(0.32-1.19)$ & \\
\hline & & & & & & & Q4 & $0.61(0.30-1.27)$ & \\
\hline & & & & & & & Q5 & $1.26(0.59-2.70)$ & \\
\hline \multirow{4}{*}{$\begin{array}{l}\text { Hansson, } 1994 \\
\text { [24], Sweden }\end{array}$} & \multirow{4}{*}{$\begin{array}{l}338(40-79 \\
\text { years/histologically } \\
\text { confirmed) }\end{array}$} & \multirow{4}{*}{$\begin{array}{l}679 \\
\text { population- } \\
\text { based }\end{array}$} & \multirow{4}{*}{ 1989-1992 } & \multirow{4}{*}{ 45-item FFQ } & \multirow{4}{*}{$\begin{array}{l}\text { Nitrates } \\
\text { mg/day }\end{array}$} & \multirow{4}{*}{$\begin{array}{l}\text { estimated by matching } \\
\text { FFQ food items } \\
\text { (considered loss in } \\
\text { cooked dishes)/based } \\
\text { on data from several } \\
\text { Swedish sources }\end{array}$} & 23 & 1.0 (Referent) & \multirow{4}{*}{$\begin{array}{l}\text { Age, gender, } \\
\text { ascorbic acid, } \\
\beta \text {-carotene. and } \\
\alpha \text {-tocopherol }\end{array}$} \\
\hline & & & & & & & 34 & $0.85(0.57-1.25)$ & \\
\hline & & & & & & & 45 & $0.99(0.65-1.52)$ & \\
\hline & & & & & & & 69 & $0.97(0.60-1.59)$ & \\
\hline
\end{tabular}


Table 2. Cont

\begin{tabular}{|c|c|c|c|c|c|c|c|c|c|}
\hline $\begin{array}{l}\text { First Author, } \\
\text { Year, Location }\end{array}$ & $\begin{array}{l}\text { No. of Cases } \\
\text { (Age/Definition) }\end{array}$ & $\begin{array}{l}\text { No. and Type } \\
\text { of Controls }\end{array}$ & $\begin{array}{l}\text { Study } \\
\text { Period }\end{array}$ & $\begin{array}{l}\text { Intake } \\
\text { Assessment }\end{array}$ & $\begin{array}{l}\text { Analytical } \\
\text { Category }\end{array}$ & $\begin{array}{l}\text { Definition/Nutrient } \\
\text { Content Values }\end{array}$ & $\begin{array}{l}\text { Consumption } \\
\text { Categories }\end{array}$ & $\begin{array}{l}\text { Adjusted OR } \\
(95 \% \text { CI })\end{array}$ & $\begin{array}{l}\text { Adjusted } \\
\text { Variables }\end{array}$ \\
\hline \multirow{10}{*}{$\begin{array}{l}\text { La Vecchia, } \\
1994 \text { [25], Italy }\end{array}$} & \multirow{10}{*}{$\begin{array}{l}723(19-74 \\
\text { years/histologically } \\
\text { confirmed) }\end{array}$} & \multirow{10}{*}{$\begin{array}{l}2024 \\
\text { hospital-based }\end{array}$} & \multirow{10}{*}{ 1985-1992 } & \multirow{10}{*}{$\begin{array}{l}\text { 29-item } \\
\text { standard } \\
\text { questionnaire }\end{array}$} & \multirow{5}{*}{$\begin{array}{l}\text { Nitrates } \\
\text { mg/die }\end{array}$} & \multirow{10}{*}{$\begin{array}{l}\text { estimated by matching } \\
\text { questionnaire food } \\
\text { items/based on Italian } \\
\text { tables of food } \\
\text { composition }\end{array}$} & 62.95 & 1.0 (Referent) & \multirow{10}{*}{$\begin{array}{l}\text { Age, sex, } \\
\text { education, } \\
\text { family history } \\
\text { of gastric } \\
\text { cancer, body } \\
\text { mass index, and } \\
\text { total energy } \\
\text { intake }\end{array}$} \\
\hline & & & & & & & 80.70 & $0.64(0.49-0.83)$ & \\
\hline & & & & & & & 96.33 & $0.50(0.38-0.67)$ & \\
\hline & & & & & & & 116.88 & $0.52(0.39-0.70)$ & \\
\hline & & & & & & & $>116.88$ & $0.43(0.32-0.59)$ & \\
\hline & & & & & \multirow{5}{*}{$\begin{array}{l}\text { Nitrites } \\
\mathrm{mg} / \text { die }\end{array}$} & & 1.91 & 1.0 (Referent) & \\
\hline & & & & & & & 2.41 & $0.98(0.72-1.33)$ & \\
\hline & & & & & & & 2.94 & $0.99(0.72-1.36)$ & \\
\hline & & & & & & & 3.64 & $1.15(0.84-1.59)$ & \\
\hline & & & & & & & $>3.64$ & $1.35(0.96-1.88)$ & \\
\hline \multirow{9}{*}{$\begin{array}{l}\text { Pobel, } 1995 \text { [26], } \\
\text { France }\end{array}$} & \multirow{9}{*}{$\begin{array}{l}92 \text { (mean: } 66.6 \text { years, } \\
\text { SD: } \\
10.4 / \text { histologically } \\
\text { confirmed) }\end{array}$} & \multirow{9}{*}{$\begin{array}{l}128 \\
\text { hospital-based }\end{array}$} & \multirow{9}{*}{ 1985-1988 } & \multirow{9}{*}{$\begin{array}{l}\text { diet history } \\
\text { questionnaire }\end{array}$} & \multirow{3}{*}{$\begin{array}{l}\text { Nitrates } \\
\text { Tertiles }\end{array}$} & \multirow{9}{*}{$\begin{array}{l}\text { derived from dairy } \\
\text { products, meat and } \\
\text { eggs, fish, flour } \\
\text { products, fruit, } \\
\text { vegetables, } \\
\text { beverages/using a } \\
\text { composition table based } \\
\text { on literature data }\end{array}$} & $\mathrm{T} 1$ & 1.0 (Referent) & \multirow{9}{*}{$\begin{array}{l}\text { Age, sex, } \\
\text { occupation and } \\
\text { total calorie } \\
\text { intake }\end{array}$} \\
\hline & & & & & & & $\mathrm{T} 2$ & $0.49(0.24-1.01)$ & \\
\hline & & & & & & & $\mathrm{T} 3$ & $0.76(0.38-1.50)$ & \\
\hline & & & & & & & T1 & 1.0 (Referent) & \\
\hline & & & & & $\begin{array}{l}\text { Nitrites } \\
\text { Tertiles }\end{array}$ & & $\mathrm{T} 2$ & $0.83(0.41-1.67)$ & \\
\hline & & & & & & & $\mathrm{T} 3$ & $0.88(0.44-1.79)$ & \\
\hline & & & & & \multirow{3}{*}{$\begin{array}{l}\text { NDMA } \\
\text { Tertiles }\end{array}$} & & $\mathrm{T} 1$ & 1.0 (Referent) & \\
\hline & & & & & & & $\mathrm{T} 2$ & $\begin{array}{l}4.13 \\
(0.93-18.27)\end{array}$ & \\
\hline & & & & & & & $\mathrm{T} 3$ & $\begin{array}{l}7.00 \\
(1.85-26.46)\end{array}$ & \\
\hline
\end{tabular}


Table 2. Cont

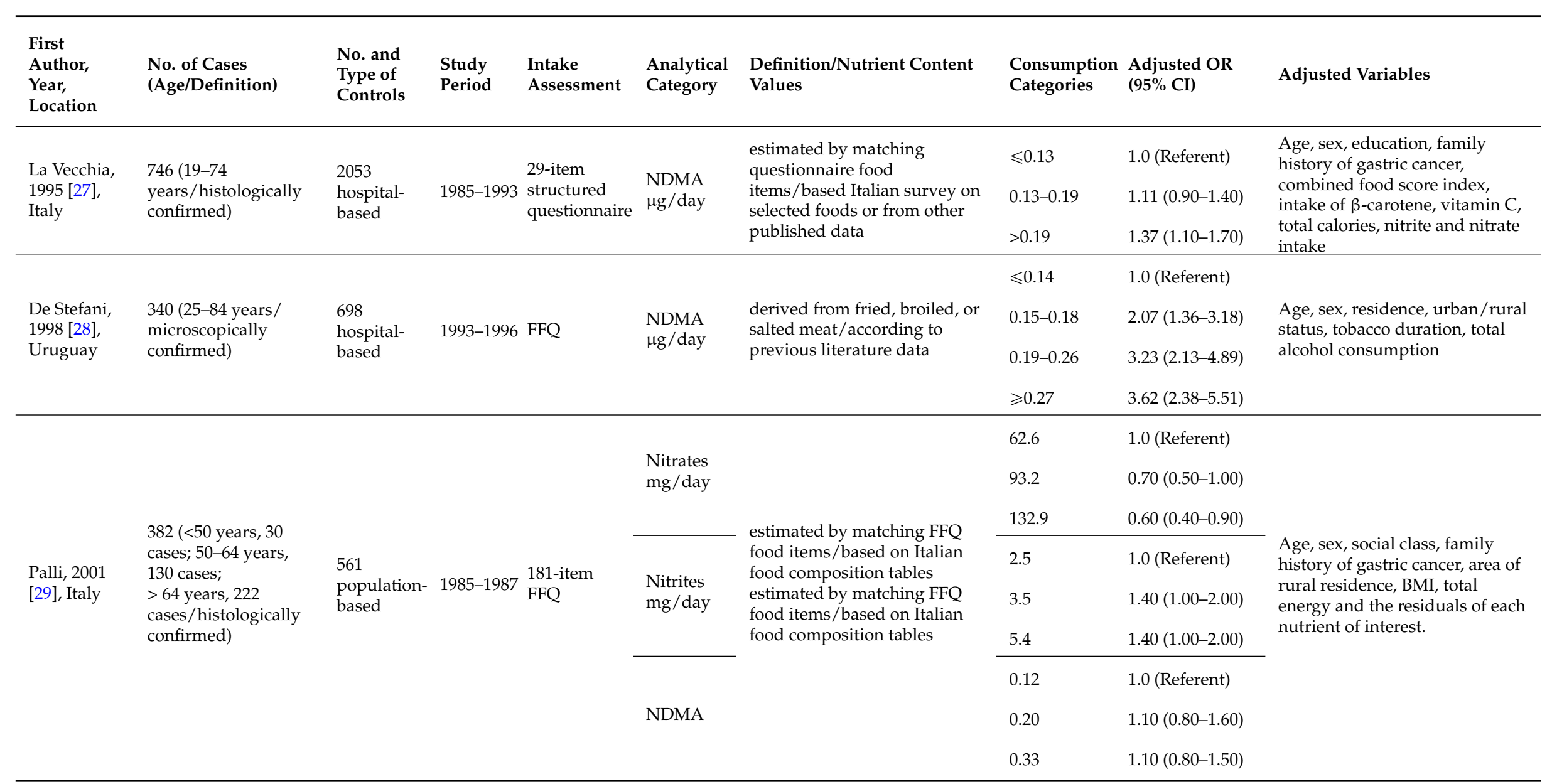


Table 2. Cont

\begin{tabular}{|c|c|c|c|c|c|c|c|c|c|}
\hline $\begin{array}{l}\text { First } \\
\text { Author, } \\
\text { Year, } \\
\text { Location }\end{array}$ & $\begin{array}{l}\text { No. of Cases } \\
\text { (Age/Definition) }\end{array}$ & $\begin{array}{l}\text { No. and } \\
\text { Type of } \\
\text { Controls }\end{array}$ & $\begin{array}{l}\text { Study } \\
\text { Period }\end{array}$ & $\begin{array}{l}\text { Intake } \\
\text { Assessment }\end{array}$ & $\begin{array}{l}\text { Analytical } \\
\text { Category }\end{array}$ & $\begin{array}{l}\text { Definition/Nutrient } \\
\text { Content Values }\end{array}$ & $\begin{array}{l}\text { Consumption } \\
\text { Categories }\end{array}$ & $\begin{array}{l}\text { Adjusted OR } \\
(95 \% \text { CI })\end{array}$ & Adjusted Variables \\
\hline $\begin{array}{l}\text { Engel, } \\
2003 \text { [30], } \\
\text { USA }\end{array}$ & $\begin{array}{l}629(30-79 \\
\text { years / histologic } \\
\text { reports from } \\
\text { surgery, } \\
\text { radiology, and } \\
\text { endoscopy) }\end{array}$ & $\begin{array}{l}695 \\
\text { population- } \\
\text { based }\end{array}$ & 1993-1995 & FFQ & $\begin{array}{l}\text { Nitrites } \\
\text { mg/day }\end{array}$ & $\begin{array}{l}\text { estimated by matching } \\
\text { FFQ food items/based } \\
\text { on a nitrite database } \\
\text { used in North America }\end{array}$ & $\begin{array}{l}\text { Men, Women } \\
1.7-5.8,1.9-5.3 \\
5.9-7.5,5.4-6.9 \\
7.6-9.9,7.0-9.1 \\
10-39.2 \\
9.2-31.2\end{array}$ & $\begin{array}{l}1.0 \text { (Referent) } \\
1.50(1.00-2.40) \\
1.80(1.10-3.00) \\
2.50(1.40-4.30)\end{array}$ & NA \\
\hline $\begin{array}{l}\text { López- } \\
\text { Carrillo, } \\
2004 \text { [31], } \\
\text { Mexico }\end{array}$ & $\begin{array}{l}211 \text { ( } \geqslant 20 \text { years / } \\
\text { histologically } \\
\text { confirmed) }\end{array}$ & $\begin{array}{l}454 \\
\text { hospital- } \\
\text { based }\end{array}$ & 1994-1996 & $\begin{array}{l}\text { semi- } \\
\text { quantitative } \\
\text { questionnaire }\end{array}$ & $\begin{array}{l}\text { Nitrites } \\
\text { portions/day }\end{array}$ & $\begin{array}{l}\text { derived from specific } \\
\text { food consumption that } \\
\text { is typical of each } \\
\text { geographical region }\end{array}$ & $\begin{array}{l}0-0.11 \\
0.12-0.26 \\
0.27-2.25\end{array}$ & $\begin{array}{l}1.0 \text { (Referent) } \\
0.95(0.62-1.46) \\
1.24(0.81-1.90)\end{array}$ & $\begin{array}{l}\text { Age, gender, residence, } \\
\text { energy change in } \\
\text { socioeconomic level, years } \\
\text { of education, Hp/CagA } \\
\text { status, and ascorbic acid }\end{array}$ \\
\hline $\begin{array}{l}\text { Kim, } 2007 \\
{[32]} \\
\text { Korea }\end{array}$ & $\begin{array}{l}136 \text { (mean: } 57.2 \\
\text { years, SD: } \\
\text { 13.9/histologically } \\
\text { confirmed) }\end{array}$ & $\begin{array}{l}136 \\
\text { hospital- } \\
\text { based }\end{array}$ & 1997-1998 & $\begin{array}{l}\text { 84-item } \\
\text { semiquantitative } \\
\text { FFQ }\end{array}$ & $\begin{array}{l}\text { Nitrates } \\
\text { mg/day }\end{array}$ & $\begin{array}{l}\text { estimated by matching } \\
\text { FFQ food items/base on } \\
\text { National Nutrition } \\
\text { Survey Report in Koera }\end{array}$ & $\begin{array}{l}240 \\
458 \\
811\end{array}$ & $\begin{array}{l}1.0 \text { (Referent) } \\
1.13(0.54-2.36) \\
1.13(0.42-3.06)\end{array}$ & $\begin{array}{l}\text { Age, sex, socioeconomic } \\
\text { status, family history, } \\
\text { refrigerator use, H. pylori } \\
\text { infection, and foods }\end{array}$ \\
\hline $\begin{array}{l}\text { Ward, } \\
2008 \text { [33], } \\
\text { USA }\end{array}$ & $\begin{array}{l}79(\geqslant 21 \\
\text { years/histologically } \\
\text { confirmed })\end{array}$ & $\begin{array}{l}321 \\
\text { population- } \\
\text { based }\end{array}$ & 1988-1994 & $\begin{array}{l}\text { short Health } \\
\text { Habits and } \\
\text { History } \\
\text { Questionnaire }\end{array}$ & $\begin{array}{l}\text { Nitrites } \\
\text { mg/day }\end{array}$ & $\begin{array}{l}\text { derived from } \\
\text { vegetables, processed } \\
\text { meats, and water/based } \\
\text { on previous published } \\
\text { literature }\end{array}$ & $\begin{array}{l}<16.9 \\
16.9-26.2 \\
26.2-38.8 \\
>38.8 \\
<0.36 \\
0.36-0.52 \\
0.52-0.67 \\
>0.67\end{array}$ & $\begin{array}{l}1.20(0.60-2.50) \\
1.40(0.70-2.90) \\
1.60(0.70-3.60) \\
1.0 \text { (Referent) } \\
1.10(0.40-2.70) \\
0.80(0.30-2.20) \\
1.10(0.30-3.40)\end{array}$ & $\begin{array}{l}\text { Year of birth, gender, } \\
\text { education, smoking, } \\
\text { alcohol, total calories, } \\
\text { vitamin C, fiber, and } \\
\text { carbohydrate }\end{array}$ \\
\hline
\end{tabular}


Table 2. Cont.

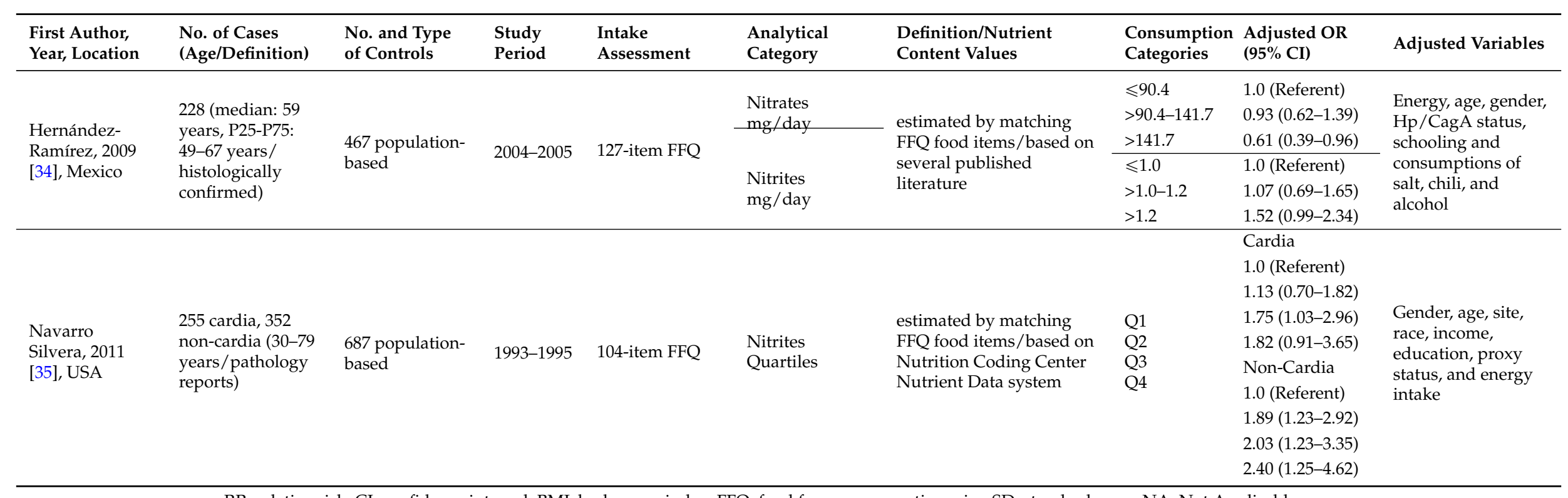

RR: relative risk; CI: confidence interval; BMI: body mass index; FFQ: food frequency questionnaire; SD: standard error; NA: Not Applicable. 


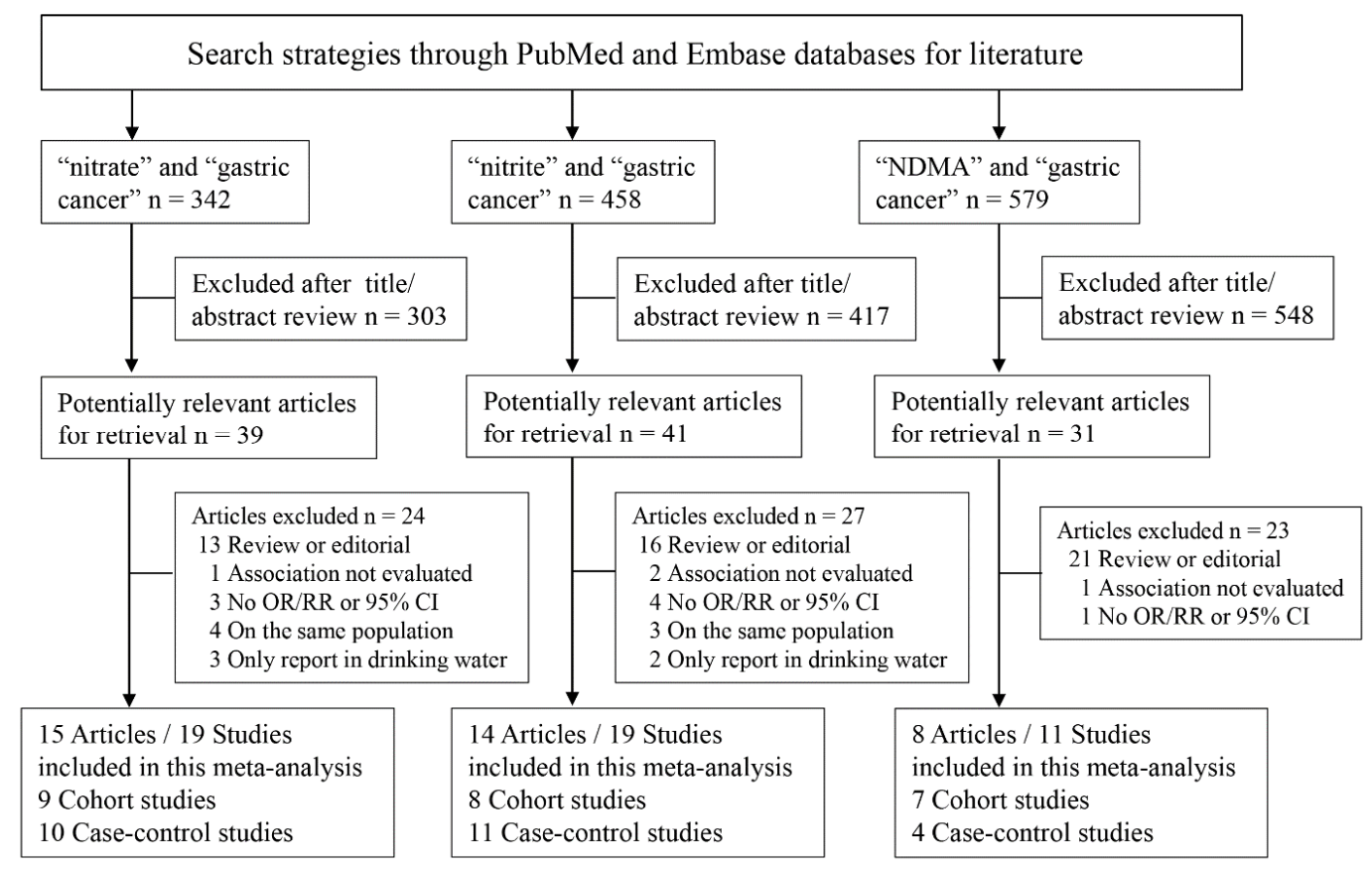

Figure 1. Flowchart of literature search and articles identified.

\subsection{Dietary Nitrates, Nitrites, and NDMA Intake and the Risk of Gastric Cancer}

There were nine cohort studies and 10 case-control studies of the relationship between the nitrates intake and gastric cancer risk [15-17,19-26,29,32-34]. Comparing the highest versus the lowest categories, the summary RR was 0.80 (95\% CI, 0.69-0.93; Figure 2A) with significant heterogeneity $\left(I^{2}=46.1 \%, p=0.015\right)$. To explore the heterogeneity, we conducted subgroup analysis according to some key characteristics. In stratified analysis by study design, significant inverse association was observed in population-based case-control studies (RR, 0.76; 95\% CI, 0.62-0.94) with acceptable heterogeneity $\left(I^{2}=47.8 \%, p=0.088\right)$. Stratifying by geographic area, the RR was $0.79(95 \% \mathrm{CI}$, 0.64-0.98) in Europe. Besides, the associations between nitrates intake and risk of stomach cancer were similar in these subgroups (publication year $<2000$, sample size $<2000$, quality score $<7$ stars; Table 3).

In pooled analysis of eight cohort studies and 10 case-control studies for nitrites $[16,17,19-22,25,26,29-31,33-35]$, a significant association was observed. Overall, individuals with highest nitrites consumption, compared with the lowest, increased the risk of gastric cancer (pooled RR, 1.31; 95\% CI, 1.13-1.52, Figure 2B). In the subgroup analysis by study design, the association was detected in both population-based case-control studies (RR, 1.72; 95\% CI, 1.47-2.02) and hospital-based case-control studies (RR, 1.25; 95\% CI, 1.09-1.44) with no heterogeneity. The risk for developing gastric cancer was significantly higher in Europe (RR: 1.30; 95\% CI, 1.12-1.50). The risk effect of nitrites was also found in subgroups (publication year, before and after 2000; sample size < 2000; quality score $<7$ stars; Table 3).

A total seven cohort studies and four case-control studies were pooled together to assess the association between NDMA consumption and stomach cancer risk $[17,18,20,26-29,36]$. The pooled RR for high versus low intake was 1.34 (95\% CI, 1.02-1.76), with obvious evidence of heterogeneity $\left(I^{2}=75.8 \%, p<0.001\right.$; Figure 2C). Study design, geographic area, cancer type, publication years, and sample size in association of NDMA consumption and gastric cancer were assessed separately. These RR estimates obtained from these subgroups showed no significant association (Table 3). Additionally, a slight association was observed in high quality studies (score $\geqslant 7$ stars; RR, 1.30; 95\% CI, 0.97-1.75). 


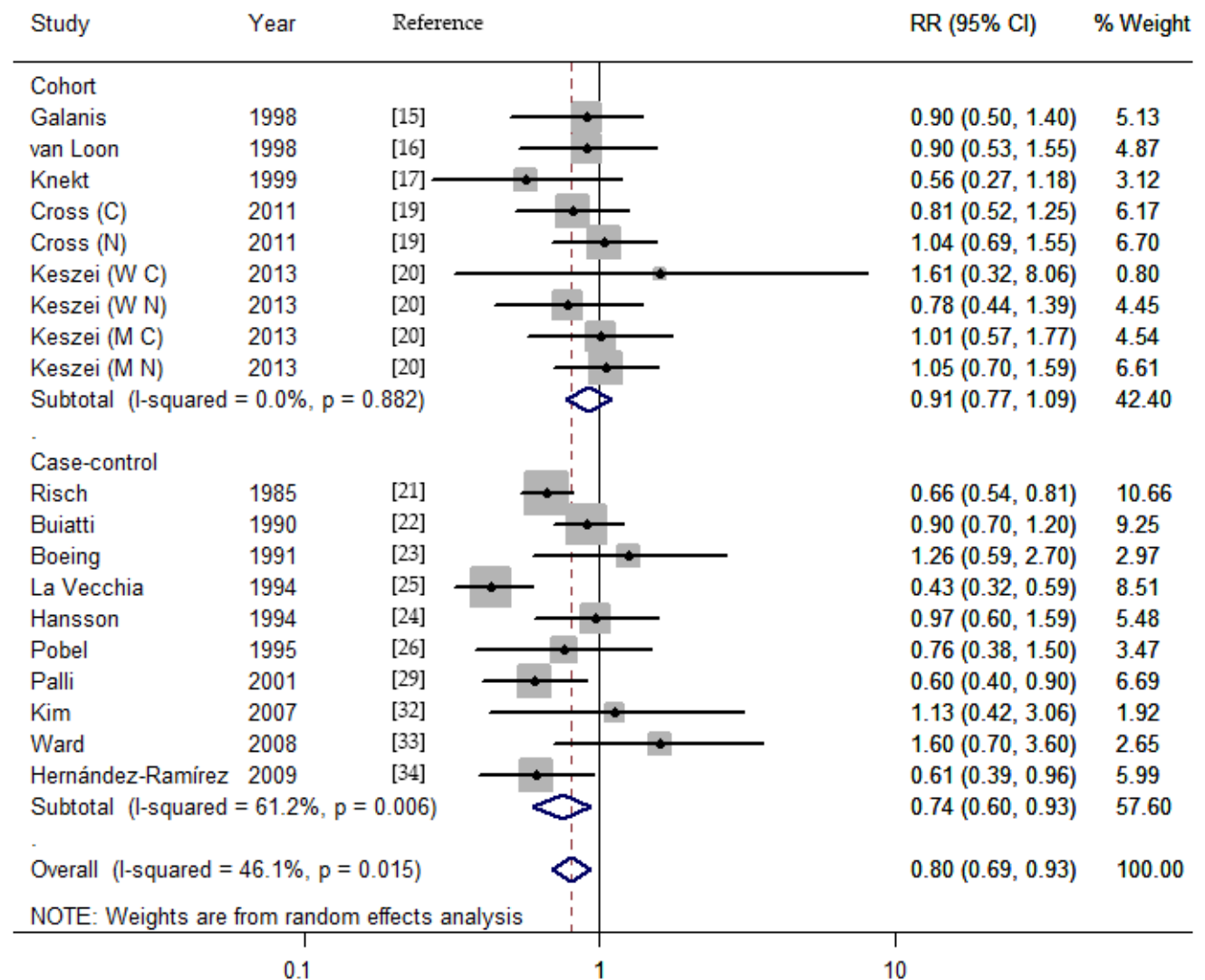

(A)

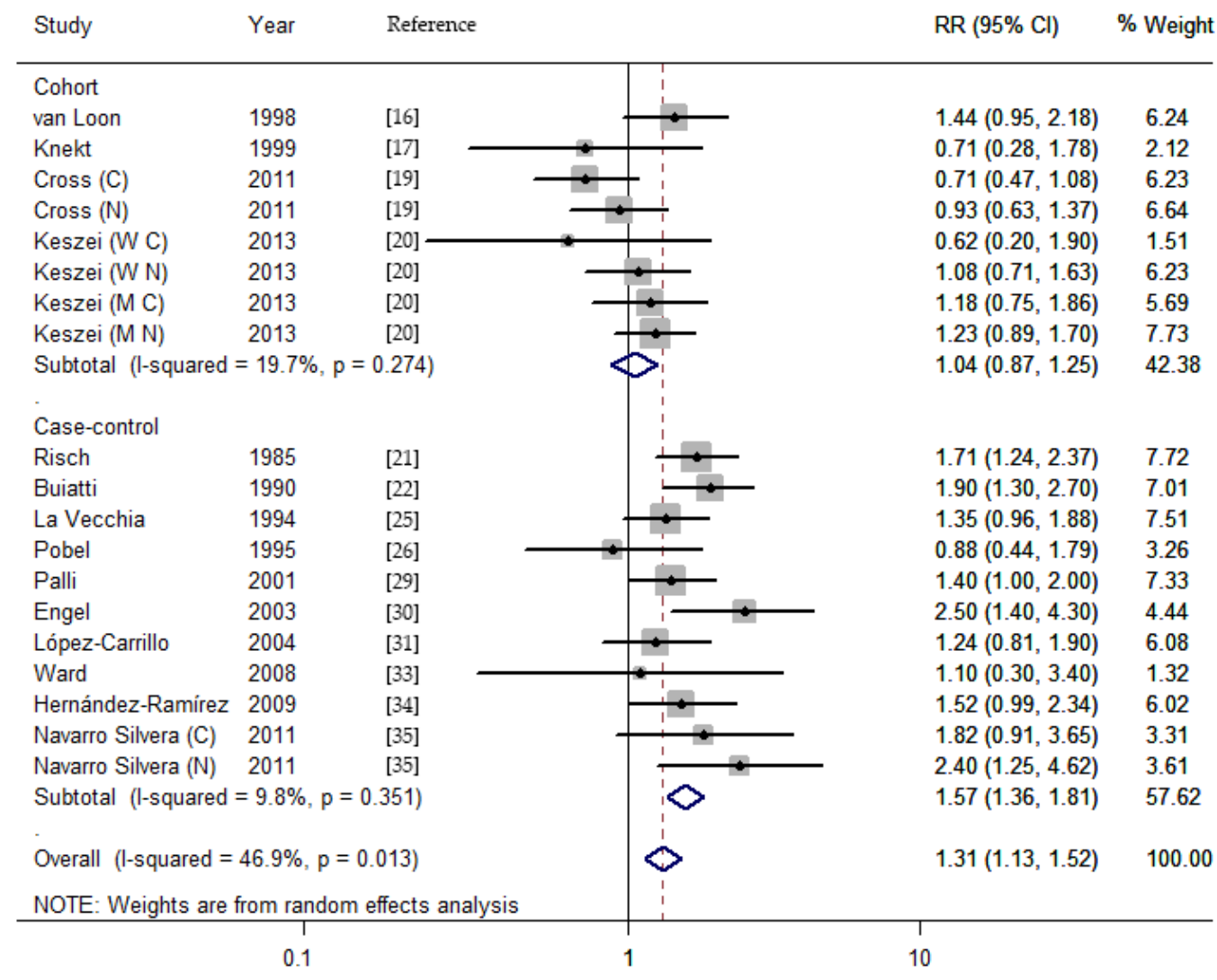

(B)

Figure 2. Cont. 


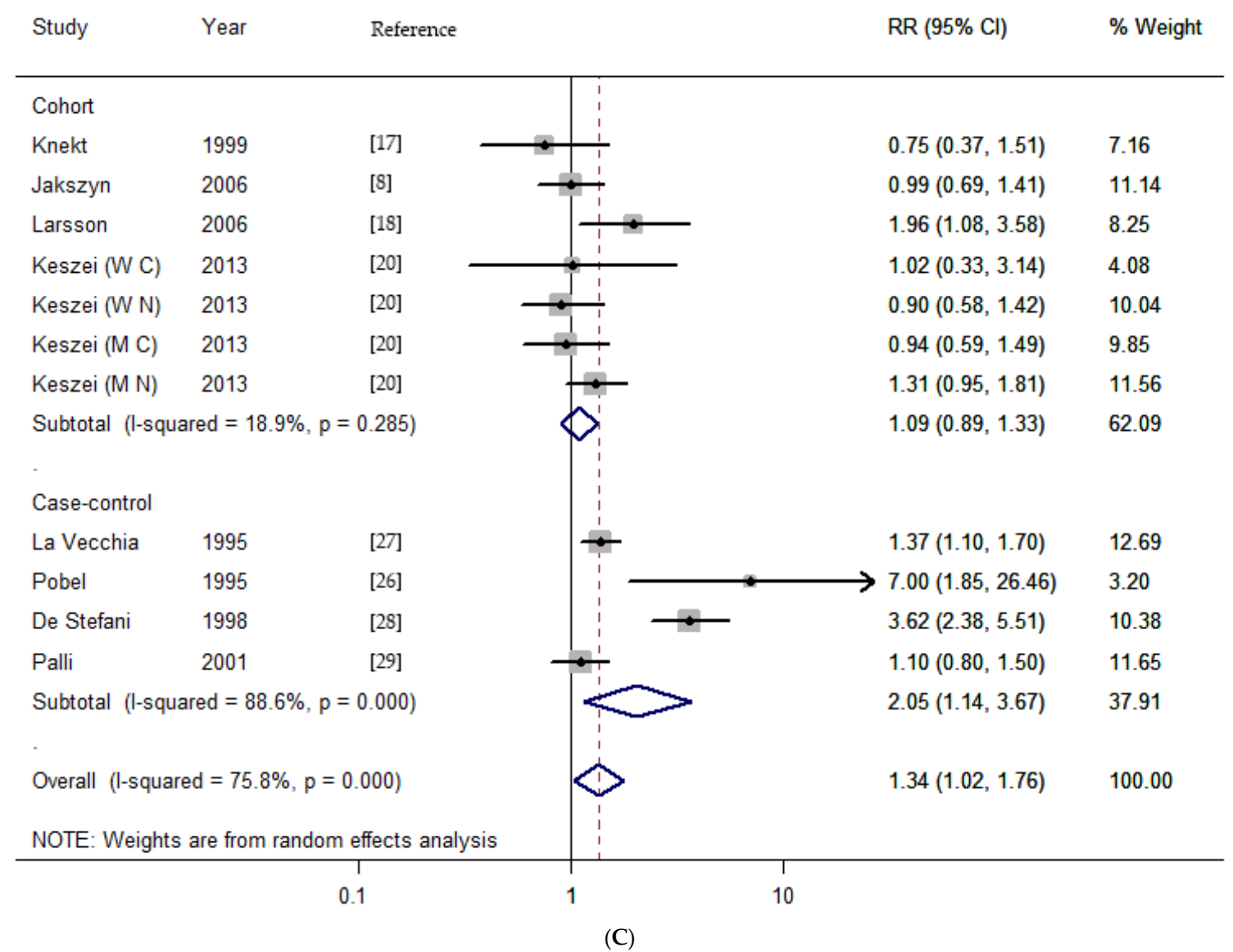

Figure 2. Dietary nitrates, nitrites and NDMA intake and the risk of gastric cancer for the highest versus lowest categories. (A) nitrates; (B) nitrites; (C) NDMA. (C, cardia; N, non-cardia; M, male; $\mathrm{W}$, women). 
Table 3. Stratified analysis of the association between nitrates, nitrites, and NDMA intake and stomach cancer risk.

\begin{tabular}{|c|c|c|c|c|c|c|c|c|c|c|c|c|c|c|c|}
\hline \multirow{3}{*}{ Variable } & \multicolumn{5}{|c|}{ Nitrates } & \multicolumn{5}{|c|}{ Nitrites } & \multicolumn{5}{|c|}{ NDMA } \\
\hline & \multirow{2}{*}{$n^{\mathrm{a}}$} & \multirow{2}{*}{ RR (95\% CI) } & \multicolumn{3}{|c|}{ Heterogeneity Test } & \multirow{2}{*}{$n^{\mathrm{a}}$} & \multirow{2}{*}{ RR (95\% CI) } & \multicolumn{3}{|c|}{ Heterogeneity Test } & \multirow{2}{*}{$n^{\mathrm{a}}$} & \multirow{2}{*}{ RR $(95 \%$ CI) } & \multicolumn{3}{|c|}{ Heterogeneity Test } \\
\hline & & & $\mathbf{Q}$ & $p^{b}$ & $I^{2 \%}$ & & & $\mathbf{Q}$ & $p^{b}$ & $I^{2 \%}$ & & & $\mathbf{Q}$ & $p^{\mathrm{b}}$ & $I^{2 \%}$ \\
\hline Total & 19 & $0.80(0.69-0.93)$ & 31.39 & 0.015 & 46.1 & 19 & $1.31(1.13-1.52)$ & 33.87 & 0.013 & 46.9 & 11 & $1.34(1.02-1.76)$ & 41.35 & $<0.001$ & 75.8 \\
\hline \multicolumn{16}{|l|}{$\begin{array}{l}\text { Study } \\
\text { design }\end{array}$} \\
\hline Cohort & 9 & $0.91(0.77-1.09)$ & 3.71 & 0.882 & 0.0 & 8 & $1.04(0.87-1.25)$ & 8.71 & 0.274 & 19.7 & 7 & $1.09(0.89-1.33)$ & 7.4 & 0.258 & 18.9 \\
\hline \multicolumn{16}{|l|}{ Case-control } \\
\hline $\begin{array}{c}\text { Population } \\
\text { based }\end{array}$ & 6 & $0.76(0.62-0.94)$ & 9.58 & 0.088 & 47.8 & 8 & $1.72(1.47-2.02)$ & 5.21 & 0.634 & 0.0 & 1 & $1.10(0.80-1.50)$ & NA & NA & NA \\
\hline $\begin{array}{c}\text { Hospital } \\
\text { based }\end{array}$ & 4 & $0.75(0.42-1.35)$ & 9.91 & 0.019 & 69.7 & 3 & $1.25(1.09-1.44)$ & 1.16 & 0.559 & 0.0 & 3 & $2.81(1.16-6.80)$ & 20.54 & $<0.001$ & 90.3 \\
\hline \multicolumn{16}{|l|}{$\begin{array}{c}\text { Geographic } \\
\text { area }\end{array}$} \\
\hline Europe & 12 & $0.79(0.64-0.98)$ & 24.03 & 0.013 & 54.2 & 10 & $1.30(1.12-1.50)$ & 10.14 & 0.339 & 11.3 & 10 & $1.18(0.97-1.43)$ & 16.89 & 0.050 & 46.7 \\
\hline $\begin{array}{l}\text { North } \\
\text { America }\end{array}$ & 5 & $0.80(0.62-1.04)$ & 8.22 & 0.084 & 51.3 & 9 & $1.41(1.06-1.87)$ & 23.62 & 0.003 & 66.1 & 0 & NA & NA & NA & NA \\
\hline Other & 2 & $0.94(0.60-1.49)$ & 0.16 & 0.690 & 0.0 & 0 & NA & NA & NA & NA & 1 & $3.62(2.38-5.51)$ & NA & NA & NA \\
\hline \multicolumn{16}{|l|}{ Cancer type } \\
\hline cardia & 3 & $0.90(0.64-1.27)$ & 0.88 & 0.644 & 0.0 & 4 & $1.01(0.65-1.58)$ & 6.64 & 0.084 & 54.8 & 3 & $0.87(0.60-1.25)$ & 0.66 & 0.718 & 0.0 \\
\hline non-cardia & 3 & $0.99(0.76-1.28)$ & 0.79 & 0.672 & 0.0 & 4 & $1.22(0.90-1.65)$ & 6.21 & 0.102 & 51.7 & 3 & $1.14(0.90-1.44)$ & 1.81 & 0.404 & 0.0 \\
\hline \multicolumn{16}{|l|}{$\begin{array}{c}\text { Publication } \\
\text { year }\end{array}$} \\
\hline$<2000$ & 9 & $0.75(0.60-0.93)$ & 19.56 & 0.012 & 59.1 & 6 & $1.46(1.17-1.81)$ & 7.38 & 0.194 & 32.3 & 4 & $2.02(0.96-4.24)$ & 25.62 & $<0.001$ & 88.3 \\
\hline$\geqslant 2000$ & 10 & $0.86(0.72-1.03)$ & 10.59 & 0.305 & 15.0 & 13 & $1.26(1.05-1.53)$ & 23.40 & 0.025 & 48.7 & 7 & $1.12(0.95-1.31)$ & 6.22 & 0.399 & 3.6 \\
\hline \multicolumn{16}{|l|}{ Sample size } \\
\hline$<2000$ & 8 & $0.76(0.62-0.94)$ & 9.99 & 0.189 & 29.9 & 9 & $1.56(1.31-1.87)$ & 9.26 & 0.321 & 13.6 & 3 & $2.69(0.95-7.60)$ & 24.06 & $<0.001$ & 91.7 \\
\hline$\geqslant 2000$ & 11 & $0.82(0.66-1.01)$ & 22.50 & 0.013 & 55.6 & 10 & $1.15(0.95-1.40)$ & 17.85 & 0.037 & 49.6 & 8 & $1.16(0.97-1.39)$ & 9.84 & 0.198 & 28.9 \\
\hline \multicolumn{16}{|l|}{$\begin{array}{l}\text { Quality } \\
\text { score }\end{array}$} \\
\hline$<7$ stars & 7 & $0.70(0.54-0.90)$ & 16.83 & 0.010 & 64.3 & 6 & $1.58(1.11-1.49)$ & 7.76 & 0.170 & 35.6 & 2 & $2.47(0.41-14.91)$ & 7.04 & 0.008 & 85.8 \\
\hline$\geqslant 7$ stars & 12 & $0.90(0.77-1.04)$ & 8.59 & 0.660 & 0.0 & 13 & $1.18(0.99-1.40)$ & 18.12 & 0.112 & 33.8 & 9 & $1.30(0.97-1.75)$ & 34.01 & $<0.001$ & 76.5 \\
\hline
\end{tabular}

RR: relative risk; CI: confidence interval; NA: Not Applicable. ${ }^{a}$ Number of comparisons; ${ }^{b} p$ Value of Q-test for heterogeneity test. 


\subsection{Dose-Response Analysis}

Four articles (7 studies) were eligible for the dose-response analysis of dietary nitrates intake and gastric cancer risk $[16,20,25,32]$. A nonlinear association was detected ( $p_{\text {non-linearity }}=0.001$, Figure 3A), with a significantly decreased risk at the nitrates intake level ranged from about 66.4 to $220 \mathrm{mg} /$ day. After evaluating the dose-response pattern for nitrites ( 2 articles / 5 studies) [16,20], some evidence of a linear association of gastric cancer was found $\left(p_{\text {linearity }}=0.041\right.$, Figure 3B). Accordingly, the summary RR for $0.1 \mathrm{mg} /$ day increment of nitrites consumption was 1.07 (95\% CI, 1.00-1.15) without heterogeneity $(p=0.876)$. Four papers (seven studies) were included in the dose-response analysis for NDMA $[18,20,27,28]$. We observed a nonlinear trend toward gastric cancer risk with increasing NDMA intake ( $\left.p_{\text {non-linearity }}<0.001\right)$, following an increase in the risk of NDMA intake up to $0.12 \mu \mathrm{g} /$ day (Figure 3C).

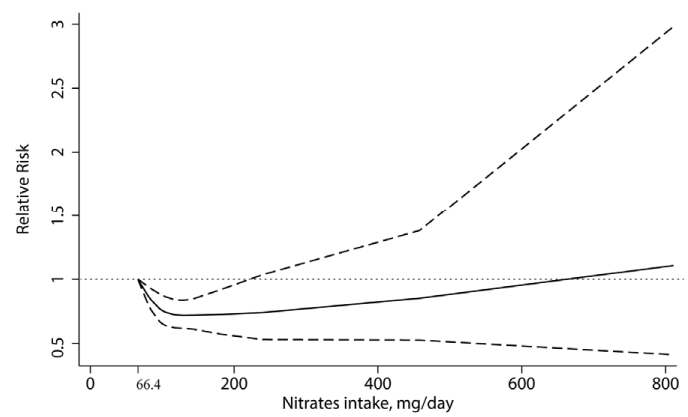

(A)

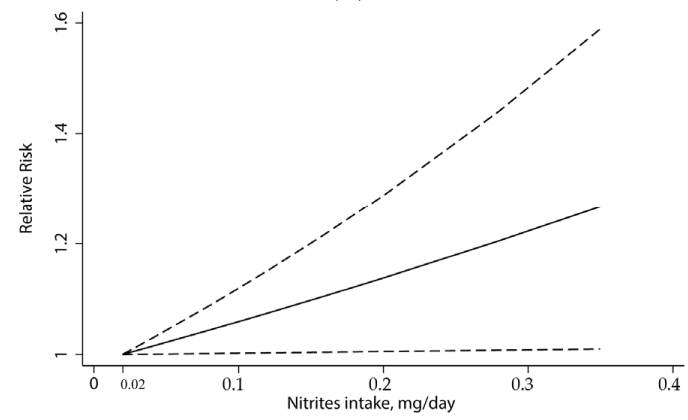

(B)

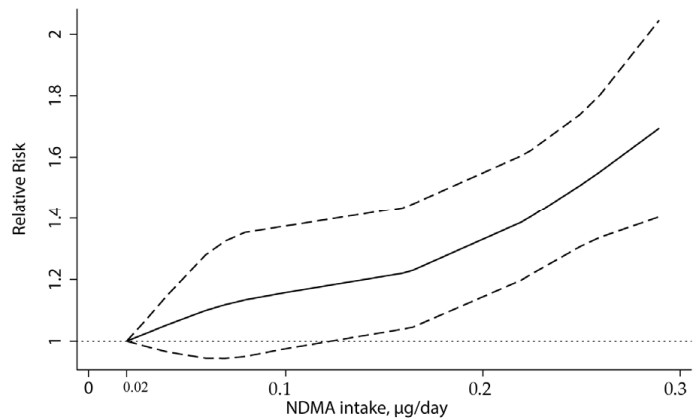

(C)

Figure 3. Dose-response analysis of dietary nitrates, nitrites and NDMA intake and the risk of gastric cancer. (A) the median value of the lowest reference interval $(66.4 \mathrm{mg} /$ day $)$ was used to estimate all relative risks for nitrates; $(\mathbf{B})$ the median value of the lowest reference interval $(0.02 \mathrm{mg} /$ day $)$ was used to estimate all relative risks for nitrites; (C) the minimum value of the lowest reference interval $(0.02 \mu \mathrm{g} /$ day $)$ was used to estimate all relative risks for NDMA. The solid line represents estimated RRs and dashed lines are their 95\% CIs. The dotted line represents the null hypothesis of no association. 


\subsection{Meta-Regression}

As shown in Table 4, study design seemed to influence the overall heterogeneity mostly for the association of nitrates intake and gastric cancer risk. In univariate meta-regression analysis, study design alone could explain $58.14 \%(0.025 / 0.043)$ of the estimated between-study variance $\left(\tau^{2}\right)$. When all the variables (study design, geographic area, and publication year) in the meta-regression model, the $\tau^{2}$ was reduced from 0.041 to 0.009 for nitrites, and from 0.139 to 0.026 for NDMA. Moreover, we found that study type was the main source of heterogeneity for nitrites, which interpreted $89.1 \%$ $(0.041 / 0.046)$ of the $\tau^{2}$. Although geographic area could explain $92.1 \%(0.128 / 0.139)$ of the $\tau^{2}$ for NDMA, the subgroups stratifying by this variable still had non-negligible heterogeneity.

Table 4. Meta-regression analysis.

\begin{tabular}{cccccccccc}
\hline Variable & \multicolumn{3}{c}{ Nitrates } & \multicolumn{3}{c}{ Nitrites } & \multicolumn{3}{c}{ NDMA } \\
\cline { 2 - 10 } & Coefficient & $p$ Value & $\mathbf{9 5 \% ~ C I}$ & Coefficient & $p$ Value & $\mathbf{9 5 \% ~ C I ~}$ & Coefficient & $p$ Value & $\mathbf{9 5 \% ~ C I ~}$ \\
\hline $\begin{array}{c}\text { Study } \\
\text { design }\end{array}$ & -0.154 & 0.184 & $\begin{array}{c}-0.390 \\
\text { to } 0.082\end{array}$ & 0.406 & 0.011 & $\begin{array}{c}0.106 \text { to } \\
0.705\end{array}$ & 0.200 & 0.363 & $\begin{array}{c}-0.286 \\
\text { to } 0.686\end{array}$ \\
\hline $\begin{array}{c}\text { Geographic } \\
\text { area }\end{array}$ & 0.023 & 0.846 & $\begin{array}{c}-0.225 \\
\text { to } 0.271\end{array}$ & -0.030 & 0.831 & $\begin{array}{c}-0.326 \\
\text { to } 0.265\end{array}$ & 0.912 & 0.057 & $\begin{array}{c}-0.035 \\
\text { to } 1.860\end{array}$ \\
\hline $\begin{array}{c}\text { Publication } \\
\text { year }\end{array}$ & 0.063 & 0.696 & $\begin{array}{c}-0.275 \\
\text { to } 0.400\end{array}$ & -0.029 & 0.845 & $\begin{array}{c}-0.343 \\
\text { to } 0.285\end{array}$ & 0.097 & 0.807 & $\begin{array}{c}-0.806 \\
\text { to } 0.999\end{array}$ \\
\hline
\end{tabular}

CI: confidence interval.

\subsection{Sensitivity Analysis}

We confirmed the associations between dietary nitrates, nitrites, and NDMA intake and gastric cancer risk were relatively stable using sensitivity analysis. After removing one study at a time, the ranges of pooled RRs were $0.67-0.97,1.10-1.57$, and 0.97-1.89 for nitrates, nitrites, and NDMA, respectively (Supplementary Figure S1). As shown in Supplementary Figure S1A, the study conducted by La Vecchia et al. seemed to cause the heterogeneity [25]. This phenomenon was verified through the Galbraith plot (Supplementary Figure S2). Exclusion of this study, the heterogeneity was not detected $\left(I^{2}=8.8 \%, p=0.349\right)$, and the summary RRs were 0.82 ( $\left.95 \% \mathrm{CI}, 0.73-0.92\right)$ in the overall study and 0.79 (95\% CI, 0.66-0.95) in the case-control study. Supplementary Figure S1C displayed that one study performed by De Stefani et al. influenced the overall pooled estimates for the association between NDMA intake and gastric cancer [28]. After this study was removed, the overall RR was $1.18\left(95 \%\right.$ CI, 0.97-1.43), with moderate heterogeneity $\left(I^{2}=46.7 \%, p=0.050\right)$.

\subsection{Publication Bias}

As shown in Figure 4, these funnel plots did not reveal obvious signs of asymmetry. Moreover, the Egger and Begg test provided statistical evidence of bias for nitrates (Egger, $p=0.047$; Begg, $p=0.327$ ), nitrites (Egger, $p=0.542$; Begg, $p=0.576$ ), and NDMA (Egger, $p=0.821$; Begg, $p=1.000$ ). Adjusting the possible publication bias for nitrates using "trim and fill" method did not influence the conclusion (RR: 0.745; 95\% CI, 0.646-0.860; Figure 4A). 


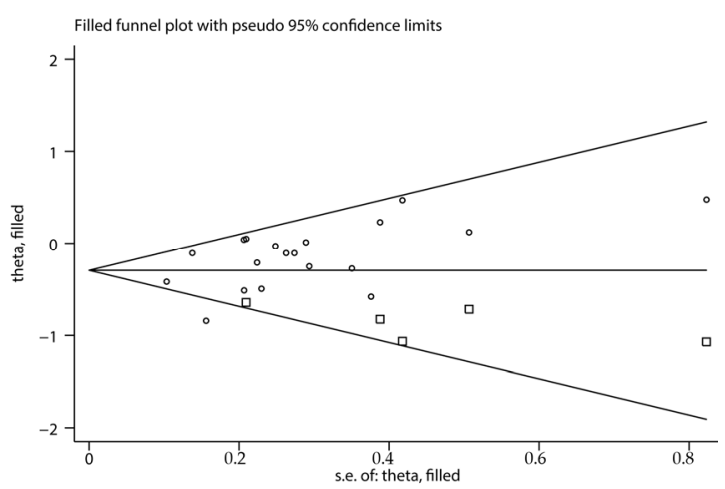

(A)

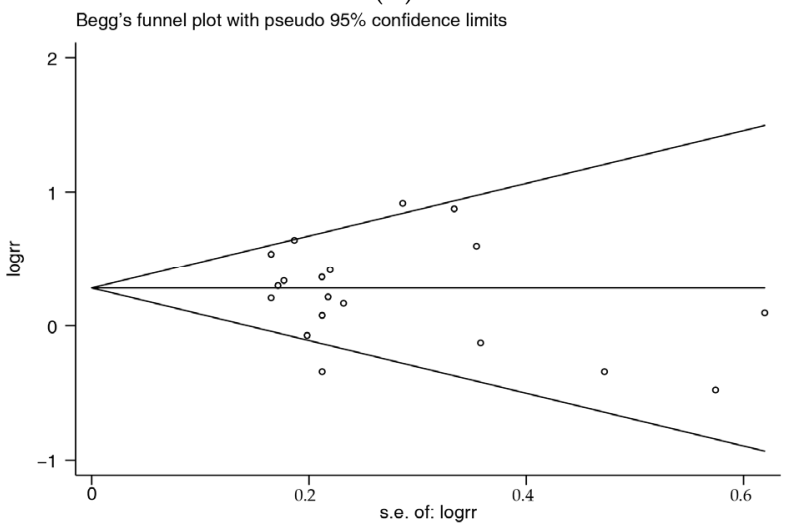

(B)

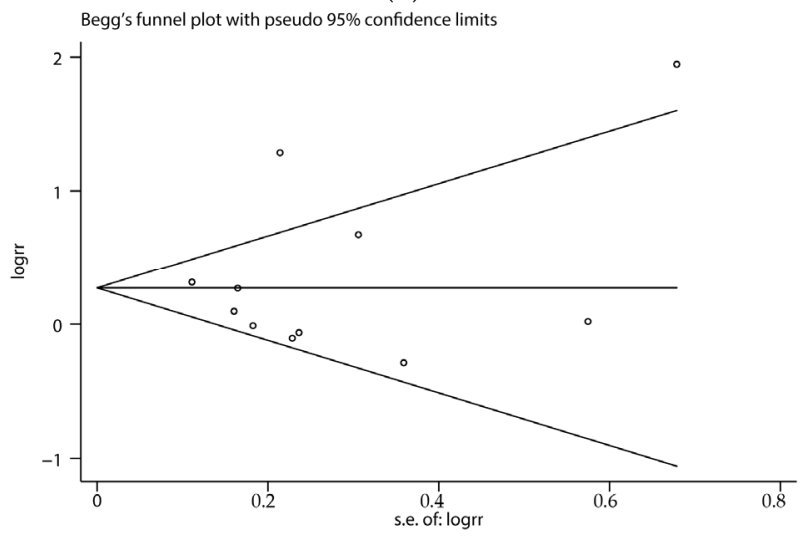

(C)

Figure 4. Funnel plot of nitrates, nitrites and NDMA consumption and gastric cancer risk. (A) Base on trim and fill method, hypothetical dummy studies indicated by squares are added to the genuine studies for nitrates; (B) nitrites; (C) NDMA.

\section{Discussion}

This is the first meat-analysis evaluating relationships between dietary nitrates, nitrites, and nitrosamines intake and the risk of gastric cancer. We found that consumption of food rich in nitrates was related to a decreased risk of gastric cancer, and that high intake of nitrites and NDMA resulted in an elevated risk of cancer. Stratifying analysis for study design, similar results were observed in the case-control studies, and the cohort studies also indicated the consequences of this trend. The dose-response analysis further showed that the inverse association between nitrates and stomach cancer appeared to be pronounced with nitrates intake level ranged from about 66.4 to $220 \mathrm{mg} /$ day. Estimated in linear dose-response model for nitrites, the risk increased in gastric cancer was 7\% 
corresponded to each $0.1 \mathrm{mg} /$ day increment of nitrites intake. When daily NDMA intake reached $0.12 \mu \mathrm{g}$, the harmful effect to human became more obvious.

In order to understand the mechanisms of nitrates, nitrites, and NDMA, we need to know its chemical and potential biologic property. Nitrates and nitrites are two types of inorganic compounds, which compose of a single nitrogen atom $(\mathrm{N})$ and a number of oxygen atoms $(\mathrm{O})$; and the chemical symbols are $\mathrm{NO}_{3}$ and $\mathrm{NO}_{2}$ for nitrate and nitrite, respectively. It is believed that nitrates themselves are relatively inert, until they are reduced to nitrites. Nitrates can turn into nitrites by bacteria in the mouth and then be swallowed. As nitrites hit the highly acidic juices in stomach, it is converted to nitrous acid, which reacts with amines to form nitrosamines [37]. In our life, processed products such as meats are heated at high temperatures, the nitrites of which can also turn into nitrosamines. Animal models were used to test the carcinogenic potential of these chemical substances. In 2010, International Agency for Research on Cancer (IARC) have concluded that there was no substantial evidence implicating nitrates as animal carcinogen [38]. Besides, nitrites in combination with amines or amides were proved to be carcinogenic to animals. Most nitrosamines can induce animal carcinogenesis by causing gene mutation and DNA adductions. Thereafter, a well-done systematic review performed by Bryan et al. elaborated the animal toxicology of these molecules and drew a consistent conclusion [39]. Whereas human diet is a potentially modifiable exposure, it remains difficult to attribute the etiology of cancer to a single nutrient. Recently, the data from prospective cohort studies, indicating that estimated intake of nitrates, nitrites, and NDMA in the diet was not significantly associated with a risk of gastric cancer $[15-17,19,20,40]$. Thus, it is necessary to conduct a meta-analysis to reveal a trend that may not be obvious in a single study.

In the present study, high nitrates consumption demonstrated a protective effect for gastric cancer, in line with some previous studies $[25,29,34]$. Because dietary nitrates are mainly provided by vegetables, and its protection is likely to be reflected by fiber, vitamin $C$, and other anti-oxidants. As is known to all, the daily intake of nitrates in Korea is highest, due to the consumption of nitrate-rich green leafy vegetables such as Kimchi, and this country is also a high-risk region for gastric cancer in Asia. Kim and his colleagues reported that the estimated values of nitrates from the Korea Food Balance Sheet (390-742 mg/day) were considerably higher, compared to European countries (52-156 mg/day) and China (422.8 mg/day). Their research also showed that a higher intake of nitrates was not related to a greater cancer risk [32]. In contrast, higher nitrates intake relative to anti-oxidants was associated with an increase the gastric cancer risk. Considering the collinearity of nitrates and antioxidant vitamins intake, we further took a meta-analysis of these studies that had adjusted for vitamin C, vegetables, or fruits, and the pooled RR was 0.97 (95\% CI, 0.81-1.17) with no heterogeneity $\left(I^{2}=0.0 \%, p=0.849\right)[16,19,20,33]$. Therefore, studies with validated methodologies quantifying the source of exposure as much as possible in the diet are needed to validate this finding.

Strengths of our meta-analysis included the large number of total subjects $(650,826$ for nitrates; 663,634 for nitrites; 742,038 for NDMA), dose-response relationship, reliable sources of heterogeneity, and the stable results in the sensitivity analysis. Here, some limitations were pointed out as follows. First, food frequency questionnaires were used to record the usual dietary consumption and classify them to estimate daily nitrates, nitrites, and NDMA intake. As a result, measurement error in different studies was inevitable, which might contribute to attenuation of the true relationship [41]. Second, there was a wide range of nitrates/nitrites/NDMA intake values between the lowest and highest categories, which might lead to the heterogeneity in the pooled analysis and conclusions limited. Third, only few articles were available for the stratified analysis of cancer type (cardia and non-cardia gastric cancer), and the dose-response analysis, especially for the nitrites (two papers / five studies), so we should treat the results with caution. More well-designed studies with detailed clinical characteristics are needed to answer these questions more completely. Fourth, significant heterogeneity was detected for NDMA, even after we confined to the stratified analysis, heterogeneity still existed in the subgroups. Fifth, Helicobacter pylori infection is a well-known risk factor for the development of distal gastric cancer. In this meta-analysis, only three case-control studies concerned 
this problem [31,32,34]. Lastly, during the long follow-up for cohort studies, the level of nitrates, nitrites, and nitrosamines in food have been marked changed due to the development of food processing technology. In addition, participants may have changed their diets and eating habits. Therefore, further prospective studies with complete questionnaires and updated diet information timely are warranted.

As diet is a very complex exposure variable, knowledge of beneficial factors and risk factors provide us an opportunity to improve heath and even prevent cancer. According to the report from WCRF/AICR [42], non-starchy vegetables as well as fruits with a relatively high content of anti-oxidants, ascorbic acid, and fiber probably protect against stomach cancer. However, salt and also salt-preserved foods have been proposed for probably causing this cancer. There is limited evidence suggesting dietary nitrates, nitrites, and NDMA intake increase the cancer risk. A review of previous research, studies of low quality tended to support the hypothesis of an increased risk with consumption of nitrite intake, while most research including better designed and conducted studies regarded NDMA as a potential human carcinogen. These results were in accord with our stratified analysis by sample size and quality score.

\section{Conclusions}

In summary, this meta-analysis suggested that dietary nitrates intake was associated with a reduced risk of gastric cancer, and high consumption of nitrites and NDMA could increase the risk. Considering the limitations and confounding factors, we could not absolutely confirm the reliability of these findings. More well-designed large prospective studies are needed to help us understand these substances in the etiology of gastric cancer.

Supplementary Materials: The following are available online at www.mdpi.com/2072-6643/7/12/5505/s1, Table S1: Methodologic quality of cohort studies included in the meta-analysis, Table S2: Methodologic quality of case-control studies included in the meta-analysis, Figure S1: Influence analysis of the summary relative risks for dietary nitrates, nitrites and NDMA intake. (A) nitrates; (B) nitrites; (C) NDMA, Figure S2: Galbraith plots of nitrates intake and gastric cancer. The central solid line and two outer parallel lines represent the estimated RRs and $95 \%$ CIs, respectively.

Acknowledgments: This work was supported by the grants from National Natural Science Foundation of China (81372364).

Conflict of Interest: The authors declare no conflict of interest.

\section{References}

1. Torre, L.A.; Bray, F.; Siegel, R.L.; Ferlay, J.; Lortet-Tieulent, J.; Jemal, A. Global cancer statistics, 2012. CA Cancer J. Clin. 2015, 65, 87-108. [CrossRef] [PubMed]

2. Abnet, C.C.; Corley, D.A.; Freedman, N.D.; Kamangar, F. Diet and upper gastrointestinal malignancies. Gastroenterology 2015, 148, 1234-1243. [CrossRef] [PubMed]

3. Petrick, J.L.; Steck, S.E.; Bradshaw, P.T.; Trivers, K.F.; Abrahamson, P.E.; Engel, L.S.; He, K.; Chow, W.H.; Mayne, S.T.; Risch, H.A.; et al. Dietary intake of flavonoids and oesophageal and gastric cancer: Incidence and survival in the United States of America (USA). Br. J. Cancer 2015, 112, 1291-1300. [CrossRef] [PubMed]

4. Bradbury, K.E.; Appleby, P.N.; Key, T.J. Fruit, vegetable, and fiber intake in relation to cancer risk: Findings from the European prospective investigation into cancer and nutrition (EPIC). Am. J. Clin. Nutr. 2014, 100 (Suppl. S1), 394S-398S. [CrossRef] [PubMed]

5. Gonzalez, C.A.; Lujan-Barroso, L.; Bueno-de-Mesquita, H.B.; Jenab, M.; Duell, E.J.; Agudo, A.; Tjonneland, A.; Boutron-Ruault, M.C.; Clavel-Chapelon, F.; Touillaud, M.; et al. Fruit and vegetable intake and the risk of gastric adenocarcinoma: A reanalysis of the European prospective investigation into cancer and nutrition (EPIC-EURGAST) study after a longer follow-up. Int. J. Cancer 2012, 131, 2910-2919. [CrossRef] [PubMed]

6. Larsson, S.C.; Orsini, N.; Wolk, A. Processed meat consumption and stomach cancer risk: A meta-analysis. J. Natl. Cancer Inst. 2006, 98, 1078-1087. [CrossRef] [PubMed] 
7. Tricker, A.R.; Pfundstein, B.; Theobald, E.; Preussmann, R.; Spiegelhalder, B. Mean daily intake of volatile $\mathrm{N}$-nitrosamines from foods and beverages in west Germany in 1989-1990. Food Chem. Toxicol. 1991, 29, 729-732. [CrossRef]

8. Jakszyn, P.; Agudo, A.; Berenguer, A.; Ibanez, R.; Amiano, P.; Pera, G.; Ardanaz, E.; Barricarte, A.; Chirlaque, M.D.; Dorronsoro, M.; et al. Intake and food sources of nitrites and N-nitrosodimethylamine in Spain. Public Health Nutr. 2006, 9, 785-791. [CrossRef] [PubMed]

9. Tricker, A.R.; Preussmann, R. Carcinogenic $N$-nitrosamines in the diet: Occurrence, formation, mechanisms and carcinogenic potential. Mutat. Res. 1991, 259, 277-289. [CrossRef]

10. Anderson, L.M.; Souliotis, V.L.; Chhabra, S.K.; Moskal, T.J.; Harbaugh, S.D.; Kyrtopoulos, S.A. $\mathrm{N}$-nitrosodimethylamine-derived $\mathrm{O}(6)$-methylguanine in DNA of monkey gastrointestinal and urogenital organs and enhancement by ethanol. Int. J. Cancer 1996, 66, 130-134. [CrossRef]

11. Stang, A. Critical evaluation of the newcastle-ottawa scale for the assessment of the quality of nonrandomized studies in meta-analyses. Eur. J. Epidemiol. 2010, 25, 603-605. [CrossRef] [PubMed]

12. Greenland, S. Quantitative methods in the review of epidemiologic literature. Epidemiol. Rev. 1987, 9, 1-30. [PubMed]

13. Orsini, N.; Li, R.; Wolk, A.; Khudyakov, P.; Spiegelman, D. Meta-analysis for linear and nonlinear dose-response relations: Examples, an evaluation of approximations, and software. Am. J. Epidemiol. 2012, 175, 66-73. [CrossRef] [PubMed]

14. Duval, S.; Tweedie, R. Trim and fill: A simple funnel-plot-based method of testing and adjusting for publication bias in meta-analysis. Biometrics 2000, 56, 455-463. [CrossRef] [PubMed]

15. Galanis, D.J.; Kolonel, L.N.; Lee, J.; Nomura, A. Intakes of selected foods and beverages and the incidence of gastric cancer among the Japanese residents of hawaii: A prospective study. Int. J. Epidemiol. 1998, 27, 173-180. [CrossRef] [PubMed]

16. Van Loon, A.J.; Botterweck, A.A.; Goldbohm, R.A.; Brants, H.A.; van Klaveren, J.D.; van den Brandt, P.A. Intake of nitrate and nitrite and the risk of gastric cancer: A prospective cohort study. Br. J. Cancer 1998, 78, 129-135. [CrossRef] [PubMed]

17. Knekt, P.; Jarvinen, R.; Dich, J.; Hakulinen, T. Risk of colorectal and other gastro-intestinal cancers after exposure to nitrate, nitrite and $N$-nitroso compounds: A follow-up study. Int. J. Cancer 1999, 80, 852-856. [CrossRef]

18. Larsson, S.C.; Bergkvist, L.; Wolk, A. Processed meat consumption, dietary nitrosamines and stomach cancer risk in a cohort of Swedish women. Int. J. Cancer 2006, 119, 915-919. [CrossRef] [PubMed]

19. Cross, A.J.; Freedman, N.D.; Ren, J.; Ward, M.H.; Hollenbeck, A.R.; Schatzkin, A.; Sinha, R.; Abnet, C.C. Meat consumption and risk of esophageal and gastric cancer in a large prospective study. Am. J. Gastroenterol. 2011, 106, 432-442. [CrossRef] [PubMed]

20. Keszei, A.P.; Goldbohm, R.A.; Schouten, L.J.; Jakszyn, P.; van den Brandt, P.A. Dietary N-nitroso compounds, endogenous nitrosation, and the risk of esophageal and gastric cancer subtypes in the Netherlands cohort study. Am. J. Clin. Nutr. 2013, 97, 135-146. [CrossRef] [PubMed]

21. Risch, H.A.; Jain, M.; Choi, N.W.; Fodor, J.G.; Pfeiffer, C.J.; Howe, G.R.; Harrison, L.W.; Craib, K.J.; Miller, A.B. Dietary factors and the incidence of cancer of the stomach. Am. J. Epidemiol. 1985, 122, 947-959. [PubMed]

22. Buiatti, E.; Palli, D.; Decarli, A.; Amadori, D.; Avellini, C.; Bianchi, S.; Bonaguri, C.; Cipriani, F.; Cocco, P.; Giacosa, A.; et al. A case-control study of gastric cancer and diet in Italy: II. Association with nutrients. Int. J. Cancer 1990, 45, 896-901. [CrossRef] [PubMed]

23. Boeing, H.; Frentzel-Beyme, R.; Berger, M.; Berndt, V.; Gores, W.; Korner, M.; Lohmeier, R.; Menarcher, A.; Mannl, H.F.; Meinhardt, M.; et al. Case-control study on stomach cancer in germany. Int. J. Cancer 1991, 47, 858-864. [CrossRef] [PubMed]

24. Hansson, L.E.; Nyren, O.; Bergstrom, R.; Wolk, A.; Lindgren, A.; Baron, J.; Adami, H.O. Nutrients and gastric cancer risk. A population-based case-control study in sweden. Int. J. Cancer 1994, 57, 638-644. [CrossRef] [PubMed]

25. La Vecchia, C.; Ferraroni, M.; D’Avanzo, B.; Decarli, A.; Franceschi, S. Selected micronutrient intake and the risk of gastric cancer. Cancer Epidemiol. Biomarkers Prev. 1994, 3, 393-398. [PubMed]

26. Pobel, D.; Riboli, E.; Cornee, J.; Hemon, B.; Guyader, M. Nitrosamine, nitrate and nitrite in relation to gastric cancer: A case-control study in marseille, france. Eur. J. Epidemiol. 1995, 11, 67-73. [CrossRef] [PubMed] 
27. La Vecchia, C.; D'Avanzo, B.; Airoldi, L.; Braga, C.; Decarli, A. Nitrosamine intake and gastric cancer risk. Eur. J. Cancer Prev. 1995, 4, 469-474. [CrossRef] [PubMed]

28. De Stefani, E.; Boffetta, P.; Mendilaharsu, M.; Carzoglio, J.; Deneo-Pellegrini, H. Dietary nitrosamines, heterocyclic amines, and risk of gastric cancer: A case-control study in Uruguay. Nutr. Cancer 1998, 30, 158-162. [CrossRef] [PubMed]

29. Palli, D.; Russo, A.; Decarli, A. Dietary patterns, nutrient intake and gastric cancer in a high-risk area of italy. Cancer Causes Control 2001, 12, 163-172. [CrossRef] [PubMed]

30. Engel, L.S.; Chow, W.H.; Vaughan, T.L.; Gammon, M.D.; Risch, H.A.; Stanford, J.L.; Schoenberg, J.B.; Mayne, S.T.; Dubrow, R.; Rotterdam, H.; et al. Population attributable risks of esophageal and gastric cancers. J. Natl. Cancer Inst. 2003, 95, 1404-1413. [CrossRef] [PubMed]

31. López-Carrillo, L.; Torres-López, J.; Galván-Portillo, M.; Muñoz, L.; López-Cervantes, M. Helicobacter pylori-caga seropositivity and nitrite and ascorbic acid food intake as predictors for gastric cancer. Eur. J. Cancer 2004, 40, 1752-1759. [CrossRef] [PubMed]

32. Kim, H.J.; Lee, S.S.; Choi, B.Y.; Kim, M.K. Nitrate intake relative to antioxidant vitamin intake affects gastric cancer risk: A case-control study in korea. Nutr. Cancer 2007, 59, 185-191. [CrossRef] [PubMed]

33. Ward, M.H.; Heineman, E.F.; Markin, R.S.; Weisenburger, D.D. Adenocarcinoma of the stomach and esophagus and drinking water and dietary sources of nitrate and nitrite. Int. J. Occup. Environ. Health 2008, 14, 193-197. [CrossRef] [PubMed]

34. Hernández-Ramírez, R.U.; Galván-Portillo, M.V.; Ward, M.H.; Agudo, A.; González, C.A.; Oñate-Ocaña, L.F.; Herrera-Goepfert, R.; Palma-Coca, O.; López-Carrillo, L. Dietary intake of polyphenols, nitrate and nitrite and gastric cancer risk in Mexico city. Int. J. Cancer 2009, 125, 1424-1430. [CrossRef] [PubMed]

35. Navarro Silvera, S.A.; Mayne, S.T.; Risch, H.A.; Gammon, M.D.; Vaughan, T.; Chow, W.H.; Dubin, J.A.; Dubrow, R.; Schoenberg, J.; Stanford, J.L.; et al. Principal component analysis of dietary and lifestyle patterns in relation to risk of subtypes of esophageal and gastric cancer. Ann. Epidemiol. 2011, 21, 543-550. [CrossRef] [PubMed]

36. Jakszyn, P.; Bingham, S.; Pera, G.; Agudo, A.; Luben, R.; Welch, A.; Boeing, H.; del Giudice, G.; Palli, D.; Saieva, C.; et al. Endogenous versus exogenous exposure to $\mathrm{N}$-nitroso compounds and gastric cancer risk in the European prospective investigation into cancer and nutrition (EPIC-EURGAST) study. Carcinogenesis 2006, 27, 1497-1501. [CrossRef] [PubMed]

37. Kobayashi, J.; Ohtake, K.; Uchida, H. No-rich diet for lifestyle-related diseases. Nutrients 2015, 7, 4911-4937. [CrossRef] [PubMed]

38. World Health Oganization; International Agency for Research on Cancer. IARC Monographs on the Evaluation of Carcinogenic Risks to Humans, Volume 94, Ingested Nitrate and Nitrite, and Cyanobacterial Peptide Toxins; International Agency for Research on Cancer: Lyon, France, 2010.

39. Bryan, N.S.; Alexander, D.D.; Coughlin, J.R.; Milkowski, A.L.; Boffetta, P. Ingested nitrate and nitrite and stomach cancer risk: An updated review. Food Chem. Toxicol. 2012, 50, 3646-3665. [CrossRef] [PubMed]

40. Loh, Y.H.; Jakszyn, P.; Luben, R.N.; Mulligan, A.A.; Mitrou, P.N.; Khaw, K.T. N-nitroso compounds and cancer incidence: The European prospective investigation into cancer and nutrition (EPIC)-norfolk study. Am. J. Clin. Nutr. 2011, 93, 1053-1061. [CrossRef] [PubMed]

41. Kipnis, V.; Subar, A.F.; Midthune, D.; Freedman, L.S.; Ballard-Barbash, R.; Troiano, R.P.; Bingham, S.; Schoeller, D.A.; Schatzkin, A.; Carroll, R.J. Structure of dietary measurement error: Results of the open biomarker study. Am. J. Epidemiol. 2003, 158, 14-21. [CrossRef] [PubMed]

42. Marmot, M.; Atinmo, T.; Byers, T.; Chen, J.; Hirohata, T.; Jackson, A.; James, W. Food, Nutrition, Physical Activity, and the Prevention of Cancer: A Global Perspective; WCRF/AICR Expert Report; World Cancer Research Fund/American Institute for Cancer Research: Washington, DC, USA, 2007.

(C) 2015 by the authors; licensee MDPI, Basel, Switzerland. This article is an open access article distributed under the terms and conditions of the Creative Commons by Attribution (CC-BY) license (http://creativecommons.org/licenses/by/4.0/). 